\title{
INTEGRABLE COUPLINGS OF SOLITON EQUATIONS BY PERTURBATIONS I. A GENERAL THEORY AND APPLICATION TO THE KDV HIERARCHY*
}

\author{
WEN-XIU MA ${ }^{\dagger}$
}

\begin{abstract}
A theory for constructing integrable couplings of soliton equations is developed by using various perturbations around solutions of perturbed soliton equations being analytic with respect to a small perturbation parameter. Multi-scale perturbations can be taken and thus higher dimensional integrable couplings can be presented. The theory is applied to the KdV soliton hierarchy. Infinitely many integrable couplings are constructed for each soliton equation in the KdV hierarchy, which contain integrable couplings possessing quadruple Hamiltonian formulations and two classes of hereditary recursion operators, and integrable couplings possessing local $2+1$ dimensional bi-Hamiltonian formulations and consequent $2+1$ dimensional hereditary recursion operators.
\end{abstract}

1. Introduction. Integrable couplings are a quite new interesting aspect in the field of soliton theory [1]. It originates from an investigation on centerless Virasoro symmetry algebras of integrable systems or soliton equations. The Abelian parts of those Virasoro symmetry algebras correspond to isospectral flows from isospectral Lax pairs and the non-Abelian parts, to non-isospectral flows from non-isospectral Lax pairs $[2,3]$. If we make a given system of soliton equations and each time part of Lax pairs of its hierarchy to be the first component and the second component of a new system respectively, then such a new system will keep the same structure of Virasoro symmetry algebras as the old one. Therefore this can lead to a hierarchy of integrable couplings for the original system.

Mathematically, the problem of integrable couplings can be expressed as: for $a$ given integrable system of evolution equations $u_{t}=K(u)$, how can we construct a nontrivial system of evolution equations which is still integrable and includes $u_{t}=K(u)$ as a sub-system?

Therefore, up to a permutation (note that we can put some components of $u_{t}=$ $K(u)$ seperately), we actually want to construct a new bigger integrable system as follows

$$
\left\{\begin{array}{l}
u_{t}=K(u) \\
v_{t}=S(u, v)
\end{array}\right.
$$

which should satisfy the non-triviality condition $\partial S / \partial[u] \neq 0$. Here $[u]$ denotes a vector consisting of all derivatives of $u$ with respect to a space variable. For example, we have $[u]=\left(u, u_{x}, u_{x x}, \cdots\right)$ in the case of $1+1$ dimensions. The non-triviality condition guarantees that trivial diagonal systems with $S(u, v)=c K(v)$ are excluded, where $c$ is an arbitrary constant.

There are two facts which have a direct relation to the study of integrable couplings. First, all possible methods for constructing integrable couplings will tell us how to extend integrable systems, from small to large and from simple to complicated, and/or how to hunt for new integrable systems, which are probably difficult to find in other ways. The corresponding theories may also provide useful information for completely classifying integrable systems in whatever dimensions. Secondly, the symmetry problem of integrable systems can be viewed as a special case of integrable

\footnotetext{
*Received September 3, 1998.

† Department of Mathematics, City University of Hong Kong, Kowloon, Hong Kong (mawx @math.cityu.edu.hk).
} 
couplings. Strictly speaking, if a system of evolution equations $u_{t}=K(u)$ is integrable, then a new system (called a perturbation system) consisting of the original system and its linearized system

$$
\left\{\begin{array}{l}
u_{t}=K(u), \\
v_{t}=K^{\prime}(u)[v],
\end{array}\right.
$$

must be still integrable [1]. The second part of the above new system is exactly the system that all symmetries need to satisfy, but new system itself is a special integrable coupling of the original system $u_{t}=K(u)$. Generally, the search for the approximate solutions $\hat{u}_{N}=\sum_{i=0}^{N} \varepsilon^{i} \eta_{i}, N \geq 1$, of physical interest to a given system $u_{t}=K(u)$ can be cast into a study of the general standard perturbation systems $\eta_{i t}=\frac{1}{i !} \frac{\partial^{i} K\left(\hat{u}_{N}\right)}{\partial \varepsilon^{i}}, 0 \leq i \leq N$. These perturbation systems were proved to form integrable couplings of the original system $u_{t}=K(u)[4,5]$, the simplest case of which is the above system associated with the symmetry problem. This fact is also a main motivation for us to consider the problem of integrable couplings.

However the standard perturbation systems above are just special examples of integrable couplings. They keep the spatial dimensions of given integrable systems invariant and only the perturbations around solutions of unperturbed integrable systems have been considered. We already know [6] that it is possible to extend the standard perturbation systems and to change the spatial dimensions, in order to make more examples of integrable couplings. The question we want to ask here is how to do generally, or what related theory we can develop. In this paper, we would like to provide our partial answer to this extensive question, by establishing a theory on the multi-scale perturbation systems of perturbed integrable systems. An approach for extending the standard perturbation systems and for enlarging the spatial dimensions by perturbations will be proposed.

Let us now introduce our basic notation and conception, some notation of which comes from Refs. $[7,8,1,5]$. Let $M=M(u)$ be a suitable manifold possessing a manifold variable $u$, which is assumed to be a column vector of $q$ functions of $t \in \mathbb{R}$ and $x \in \mathbb{R}^{p}$ with $t$ playing the role of time and $x$ representing position in space. We are concerned with coupling systems by perturbations and thus need to introduce another bigger suitable manifold $\hat{M}_{N}=\hat{M}_{N}\left(\hat{\eta}_{N}\right)$ possessing a manifold variable $\hat{\eta}_{N}=\left(\eta_{0}^{T}, \eta_{1}^{T}, \cdots, \eta_{N}^{T}\right)^{T}, N \geq 0$, where $\eta_{i}, 0 \leq i \leq N$, are also assumed to be column function vectors of the same dimension as $u$ and $T$ means the transpose of matrices. Assume that $T(M), T\left(\hat{M}_{N}\right)$ denote the tangent bundles on $M$ and $\hat{M}_{N}$, $T^{*}(M), T^{*}\left(\hat{M}_{N}\right)$ denote the cotangent bundles on $M$ and $\hat{M}_{N}$, and $C^{\infty}(M), C^{\infty}\left(\hat{M}_{N}\right)$ denote the spaces of smooth functionals on $M$ and $\hat{M}_{N}$, respectively. Moreover let $T_{s}^{r}(M)$ be the s-times co- and r-times contravariant tensor bundle and $\left.T_{s}^{r}\right|_{u}(M)$, the space of s-times co- and r-times contravariant tensors at $u \in M$. We use $X(u)$ (not $\left.\left.X\right|_{u}\right)$ to denote a tensor of $X \in T_{s}^{r}(M)$ at $u \in M$ but sometimes we omit the point $u$ for brevity if there is no confusion. Note that four linear operators $\Phi: T(M) \rightarrow T(M)$, $\Psi: T^{*}(M) \rightarrow T^{*}(M), J: T^{*}(M) \rightarrow T(M), \Theta: T(M) \rightarrow T^{*}(M)$ can be identified with the second-degree tensor fields $T_{\Phi} \in T_{1}^{1}(M), T_{\Psi} \in T_{1}^{1}(M), T_{J} \in T_{0}^{2}(M), T_{\Theta} \in$ $T_{2}^{0}(M)$ by the following relations

$$
\begin{aligned}
& T_{\Phi}(u)(\alpha(u), K(u))=<\alpha(u), \Phi(u) K(u)>, \alpha \in T^{*}(M), K \in T(M), \\
& T_{\Psi}(u)(\alpha(u), K(u))=<\Psi(u) \alpha(u), K(u)>, \alpha \in T^{*}(M), K \in T(M), \\
& T_{J}(u)(\alpha(u), \beta(u))=<\alpha(u), J(u) \beta(u)>, \alpha, \beta \in T^{*}(M), \\
& T_{\Theta}(u)(K(u), S(u))=<\Theta(u) K(u), S(u)>, K, S \in T(M),
\end{aligned}
$$


where $\langle\cdot, \cdot\rangle$ denotes the duality between cotangent vectors and tangent vectors.

Of fundamental importance is the conception of the Gateaux derivative, which provides a tool to handle various tensor fields. For a tensor field $X \in T_{s}^{r}(M)$, its Gateaux derivative at a direction $Y \in T(M)$ is defined by

$$
X^{\prime}(u)[Y(u)]=\left.\frac{\partial X(u+\varepsilon Y(u))}{\partial \varepsilon}\right|_{\varepsilon=0} .
$$

For those operators between the tangent bundle and the cotangent bundle, their Gateaux derivatives can be given similarly or by means of their tensor fields. The commutator of two vector fields $K, S \in T(M)$ and the adjoint $\operatorname{map} \operatorname{ad}_{K}: T(M) \rightarrow$ $T(M)$ are commonly defined by

$$
[K, S](u)=K^{\prime}(u)[S(u)]-S^{\prime}(u)[K(u)], \operatorname{ad}_{K} S=[K, S] .
$$

Note that there are some authors who use the other commutator

$$
[K, S](u)=S^{\prime}(u)[K(u)]-K^{\prime}(u)[S(u)]
$$

It doesn't matter of course but each type has many proponents and hence one must be careful of plus and minus signs in reading various sources.

The conjugate operators of operators between the tangent bundle and the cotangent bundle are determined in terms of the duality between cotangent vectors and tangent vectors. For instance, the conjugate operator $\Phi^{\dagger}: T^{*}(M) \rightarrow T^{*}(M)$ of an operator $\Phi: T(M) \rightarrow T(M)$ is established by

$$
<\Phi^{\dagger}(u) \alpha(u), K(u)>=<\alpha(u), \Phi(u) K(u)>, \alpha \in T^{*}(M), K \in T(M) .
$$

If an operator $J: T^{*}(M) \rightarrow T(M)$ (or $\Theta: T(M) \rightarrow T^{*}(M)$ ) plus its conjugate operator is equal to zero, then it is called to be skew-symmetric.

DeFinition 1.1. For a functional $\tilde{H} \in C^{\infty}(M)$, its variational derivative $\frac{\delta \tilde{H}}{\delta u} \in$ $T^{*}(M)$ is defined by

$$
<\frac{\delta \tilde{H}}{\delta u}(u), K(u)>=\tilde{H}^{\prime}(u)[K(u)], K \in T(M) .
$$

If for $\gamma \in T^{*}(M)$ there exists a functional $\tilde{H} \in C^{\infty}(M)$ so that

$$
\frac{\delta \tilde{H}}{\delta u}=\gamma, \text { i.e., } \tilde{H}^{\prime}(u)[K(u)]=<\gamma(u), K(u)>, K \in T(M),
$$

then $\gamma \in T^{*}(M)$ is called a gradient field with a potential $\tilde{H}$.

A cotangent vector field $\gamma \in T^{*}(M)$ is a gradient field if and only if

$$
\begin{aligned}
& (d \gamma)(u)(K(u), S(u)) \\
:= & <\gamma^{\prime}(u)[K(u)], S(u)>-<\gamma^{\prime}(u)[S(u)], K(u)>=0, K, S \in T(M) .
\end{aligned}
$$

If $\gamma \in T^{*}(M)$ is gradient, then its potential $\tilde{H}$ is given by

$$
\tilde{H}(u)=\int_{0}^{1}<\gamma(\lambda u), u>d \lambda .
$$


Definition 1.2. For a linear operator $\Phi: T(M) \rightarrow T(M)$ and a vector field $K \in T(M)$, the Lie derivative $L_{K} \Phi: T(M) \rightarrow T(M)$ of $\Phi$ with respect to $K$ is defined by

$$
\begin{aligned}
& \left(L_{K} \Phi\right)(u) S(u) \\
& =\Phi^{\prime}(u)[K(u)] S(u)-K^{\prime}(u)[\Phi(u) S(u)]+\Phi(u) K^{\prime}(u)[S(u)], \quad S \in T(M) .
\end{aligned}
$$

An equivalent form of the Lie derivative is

$$
\left(L_{K} \Phi\right)(u) S(u)=\Phi(u)[K(u), S(u)]-[K(u), \Phi(u) S(u)],
$$

where $\Phi: T(M) \rightarrow T(M), K, S \in T(M)$, and the commutator $[\cdot, \cdot]$ is defined by (1.3).

Definition 1.3. A linear operator $\Phi: T(M) \rightarrow T(M)$ is called a recursion operator of $u_{t}=K(u), K \in T(M)$, if for all $S \in T(M)$ and $u \in M$, we have

$$
\frac{\partial \Phi(u)}{\partial t} S(u)+\Phi^{\prime}(u)[K(u)] S(u)-K^{\prime}(u)[\Phi(u) S(u)]+\Phi(u) K^{\prime}(u)[S(u)]=0 .
$$

Obviously a recursion operator $\Phi: T(M) \rightarrow T(M)$ of a system $u_{t}=K(u), K \in$ $T(M)$, transforms a symmetry into another symmetry of the same system $u_{t}=K(u)$. Therefore it is very useful in constructing the corresponding symmetry algebra of a given system and its existence is regarded as an important characterizing property for integrability of the system under study.

Definition 1.4. A linear operator $\Phi: T(M) \rightarrow T(M)$ is called a hereditary operator or to be hereditary [9], if the following equality holds

$$
\begin{aligned}
& \Phi^{\prime}(u)[\Phi(u) K(u)] S(u)-\Phi(u) \Phi^{\prime}(u)[K(u)] S(u) \\
& -\Phi^{\prime}(u)[\Phi(u) S(u)] K(u)+\Phi(u) \Phi^{\prime}(u)[S(u)] K(u)=0
\end{aligned}
$$

for all vector fields $K, S \in T(M)$.

For a linear operator $\Phi: T(M) \rightarrow T(M)$, the above equality (1.8) can be replaced with either of the following equalities:

$$
\begin{aligned}
& \left(L_{\Phi K}\right)(u) \Phi(u)=\Phi(u)\left(L_{K} \Phi\right)(u), K \in T(M), \\
& \Phi^{2}(u)[K(u), S(u)]+[\Phi(u) K(u), \Phi(u) S(u)] \\
& \quad-\Phi(u)\{[K(u), \Phi(u) S(u)]+[\Phi(u) K(u), S(u)]\}=0, K, S \in T(M) .
\end{aligned}
$$

It follows directly from (1.6) that these two equalities are equivalent to each other. We point out that hereditary operators have two remarkable properties. First, if $\Phi$ : $T(M) \rightarrow T(M)$ is hereditary and $L_{K} \Phi=0, K \in T(M)$, then we have $\left[\Phi^{m} K, \Phi^{n} K\right]=$ $0, m, n \geq 0$ (see, for example, $[9,10,11]$ ). Therefore, when a system $u_{t}=K(u), K \in$ $T(M)$, possesses a time-independent hereditary recursion operator $\Phi: T(M) \rightarrow$ $T(M)$, a hierarchy of vector fields $\Phi^{n} K, n \geq 0$, are all symmetries and commute with each other. Secondly, if the conjugate operator $\Psi=\Phi^{\dagger}$ of a hereditary operator $\Phi: T(M) \rightarrow T(M)$ maps a gradient field $\gamma \in T^{*}(M)$ into another gradient field, then $\Psi^{n} \gamma, n \geq 0$, are all gradient fields (see, for example, [12]). 
Definition 1.5. A linear skew-symmetric operator $J: T^{*}(M) \rightarrow T(M)$ is called a Hamiltonian operator or to be Hamiltonian, if for all $\alpha, \beta, \gamma \in T^{*}(M)$, we have

$$
<\alpha, J^{\prime}(u)[J(u) \beta] \gamma>+\operatorname{cycle}(\alpha, \beta, \gamma)=0 .
$$

Its Poisson bracket is defined by

$$
\left\{\tilde{H}_{1}, \tilde{H}_{2}\right\}_{J}(u)=<\frac{\delta \tilde{H}_{1}}{\delta u}(u), J(u) \frac{\delta \tilde{H}_{2}}{\delta u}(u)>,
$$

where $\tilde{H}_{1}, \tilde{H}_{2} \in C^{\infty}(M)$. A pair of operators $J, M: T^{*}(M) \rightarrow T(M)$ is called a Hamiltonian pair, if $c J+d M$ is always Hamiltonian for any constants $c, d$.

When $J: T^{*}(M) \rightarrow T(M)$ is Hamiltonian, we have [13]

$$
J(u) \frac{\delta\left\{\tilde{H}_{1}, \tilde{H}_{2}\right\}_{J}}{\delta u}(u)=\left[J(u) \frac{\delta \tilde{H}_{1}}{\delta u}(u), J(u) \frac{\delta \tilde{H}_{2}}{\delta u}(u)\right],
$$

where $\tilde{H}_{1}, \tilde{H}_{2} \in C^{\infty}(M)$. This implies that the operator $J \frac{\delta}{\delta u}$ is a Lie homomorphism from the Poisson algebra to the vector field algebra. Moreover if $J, M: T^{*}(M) \rightarrow$ $T(M)$ is a Hamiltonian pair and $J$ is invertible, then $\Phi=M J^{-1}: T(M) \rightarrow T(M)$ defines a hereditary operator $[13,7]$.

Definition 1.6. A linear skew-symmetric operator $\Theta: T(M) \rightarrow T^{*}(M)$ is called a symplectic operator or to be symplectic, if for all $K, S, T \in T(M)$, we have

$$
<K(u), \Theta^{\prime}(u)[S(u)] T(u)>+\operatorname{cycle}(K, S, T)=0 .
$$

If $\Theta: T(M) \rightarrow T^{*}(M)$ is a symplectic operator, then its second-degree tensor field $T_{\Theta} \in T_{2}^{0}(M)$ can be expressed as

$$
T_{\Theta}=d \gamma \text { with }\left\langle\gamma(u), K(u)>=\int_{0}^{1}<\Theta(\lambda u) \lambda u, K(u)>d \lambda, K \in T(M),\right.
$$

where $d \gamma$ is determined by (1.4). It is not difficult to verify that the inverse of a symplectic operator is Hamiltonian if it exists and vice versa. We also mention that Hamiltonian and symplectic operators can be defined only in terms of the Dirac structures [14].

Definition 1.7. A system of evolution equations $u_{t}=K(u), K \in T(M)$, is called a Hamiltonian system or to be Hamiltonian, if there exists a functional $\tilde{H} \in$ $C^{\infty}(M)$ so that

$$
u_{t}=K(u)=J(u) \frac{\delta \tilde{H}}{\delta u}(u)
$$

It is called a bi-Hamiltonian system, if there exist two functionals $\tilde{H}_{1}, \tilde{H}_{2} \in C^{\infty}(M)$ and a Hamiltonian pair $J, M: T^{*}(M) \rightarrow T(M)$ so that

$$
u_{t}=K(u)=J(u) \frac{\delta \tilde{H}_{1}}{\delta u}(u)=M(u) \frac{\delta \tilde{H}_{2}}{\delta u}(u) .
$$


There is the other kind of Hamiltonian systems, which can be defined by symplectic operators. However, the above definition has more advantages in handling symmetries and conserved functionals. For a Hamiltonian system $u_{t}=J(u) \frac{\delta \tilde{H}}{\delta u}(u)$, the linear operator $J \frac{\delta}{\delta u}$ maps a conserved functional into a symmetry. For a bi-Hamiltonian system, we will be able to recursively construct infinitely many commuting symmetries and conserved functionals for the system, if either of two Hamiltonian operators is invertible [15].

In what follows, we would like to develop a theory for constructing integrable couplings of soliton equations, by analyzing integrable properties of the perturbation systems resulted from perturbed soliton equations by multi-scale perturbations. The paper is organized as follows. In Section 2, we first establish general explicit structures of hereditary operators, Hamiltonian operators and symplectic operators under the multi-scale perturbations. We will go on to show that the perturbations preserves complete integrability, by establishing various integrable properties of the resulting perturbation systems, such as hereditary recursion operator structures, Virasoro symmetry algebras, Lax representations, zero curvature representations, Hamiltonian formulations and so on. In Section 3, the whole theory will be applied to the KdV equations as illustrative examples. This leads to infinitely many integrable couplings of the KdV equations, which include Hamiltonian integrable couplings possessing two different hereditary recursion operators and local bi-Hamiltonian integrable couplings in $2+1$ dimensions. Finally, some concluding remarks are given in Section 5 .

\section{Integrable couplings by perturbations.}

2.1. Triangular systems by perturbations. Let us take a perturbation series for any $N \geq 0$ and $r \geq 0$ :

$$
\hat{u}_{N}=\sum_{i=0}^{N} \varepsilon^{i} \eta_{i}, \eta_{i}=\eta_{i}\left(y_{0}, y_{1}, y_{2}, \cdots, y_{r}, t\right), y_{i}=\varepsilon^{i} x, t \in \mathbb{R}, x \in \mathbb{R}^{p}, 0 \leq i \leq N
$$

where $\varepsilon$ is a perturbation parameter and $\eta_{i}, 0 \leq i \leq N$, are assumed to be column vectors of $q$ dimensions as before. When $r \geq 1,(2.1)$ is really a multi-scale perturbation series. We fix a perturbed vector field $K=K(\varepsilon) \in T(M)$ which is required to be analytic with respect to $\varepsilon$. Let us introduce

$$
K^{(i)}=K^{(i)}\left(\hat{\eta}_{N}\right)=(K(u, \varepsilon))^{(i)}\left(\hat{\eta}_{N}\right)=\left.\frac{1}{i !} \frac{\partial^{i} K\left(\hat{u}_{N}, \varepsilon\right)}{\partial \varepsilon^{i}}\right|_{\varepsilon=0}, 0 \leq i \leq N,
$$

where $\hat{\eta}_{N}=\left(\eta_{0}^{T}, \eta_{1}^{T}, \cdots, \eta_{N}^{T}\right)^{T}$ as before, and then define the $N$-th order perturbation vector field on $\hat{M}_{N}$ :

$$
\left(\operatorname{per}_{N} K\right)\left(\hat{\eta}_{N}\right)=\hat{K}_{N}\left(\hat{\eta}_{N}\right)=\left(K^{(0) T}\left(\hat{\eta}_{N}\right), K^{(1) T}\left(\hat{\eta}_{N}\right), \cdots, K^{(N) T}\left(\hat{\eta}_{N}\right)\right)^{T} .
$$

Here the vector fields on $M$ are viewed as column vectors of $q$ dimensions, and the vector fields on $\hat{M}_{N}$, column vectors of $q(N+1)$ dimensions, as they are normally handled. Since we have

$$
\left.\frac{\partial^{i} K\left(\hat{u}_{i}, \varepsilon\right)}{\partial \varepsilon^{i}}\right|_{\varepsilon=0}=\left.\frac{\partial^{i} K\left(\hat{u}_{j}, \varepsilon\right)}{\partial \varepsilon^{i}}\right|_{\varepsilon=0}, \hat{u}_{i}=\sum_{k=0}^{i} \varepsilon^{k} \eta_{k}, \hat{u}_{j}=\sum_{k=0}^{j} \varepsilon^{k} \eta_{k}, 0 \leq i \leq j \leq N
$$


it is easy to find that

$$
\begin{aligned}
& \hat{K}_{N}\left(\hat{\eta}_{N}\right)=\left(K^{(0) T}\left(\hat{\eta}_{0}\right), K^{(1) T}\left(\hat{\eta}_{1}\right), \cdots, K^{(N) T}\left(\hat{\eta}_{N}\right)\right)^{T} \\
& \hat{\eta}_{i}=\left(\eta_{0}^{T}, \eta_{1}^{T}, \cdots, \eta_{i}^{T}\right)^{T}, \quad 0 \leq i \leq N .
\end{aligned}
$$

Thus the perturbation vector field $\operatorname{per}_{N} K=\hat{K}_{N} \in T\left(\hat{M}_{N}\right)$ has a specific property that the $i$-th component depends only on $\eta_{0}, \eta_{1}, \cdots, \eta_{i}$, but not on any $\eta_{j}, j>i$.

Let us now consider a system of perturbed evolution equations

$$
u_{t}=K(u, \varepsilon), K=K(\varepsilon) \in T(M),
$$

where $K(\varepsilon)$ is assumed to be analytic with respect to $\varepsilon$, as an initial system that we start from. It is obvious that the following perturbed system

$$
\hat{u}_{N t}=K\left(\hat{u}_{N}, \varepsilon\right)+\mathrm{o}\left(\varepsilon^{N}\right) \text { or } \hat{u}_{N t} \equiv K\left(\hat{u}_{N}, \varepsilon\right) \quad\left(\bmod \varepsilon^{N}\right),
$$

leads equivalently to a bigger system of evolution equations

$$
\hat{\eta}_{N t}=\hat{K}_{N}\left(\hat{\eta}_{N}\right) \text {, i.e., } \eta_{i t}=\left.\frac{1}{i !} \frac{\partial^{i} K\left(\hat{u}_{N}, \varepsilon\right)}{\partial \varepsilon^{i}}\right|_{\varepsilon=0}, 0 \leq i \leq N,
$$

where $\hat{u}_{N}$ is defined by (2.1). Conversely, a solution $\hat{\eta}_{N}$ of the bigger system (2.7) gives rise to an approximate solution $\hat{u}_{N}$ of the initial system (2.5) to a precision $\mathrm{o}\left(\varepsilon^{N}\right)$. The resulting bigger system (2.7) is called an $N$-th order perturbation system of the initial perturbed system (2.5), and it is a triangular system, owing to (2.4). We will analyze its integrable properties by exposing structures of other perturbation objects.

2.2. Symmetry problem. Let us shed more right on an remarkable relation between the symmetry problem and integrable couplings. Assume that a system $u_{t}=K(u), K \in T(M)$, is given. Then its linearized system reads as $v_{t}=K^{\prime}(u)[v]$. What the symmetry problem requires to do is to find vector fields $S \in T(M)$ which satisfy this linearized system, i.e., $(S(u))_{t}=K^{\prime}(u)[S(u)]$ when $u_{t}=K(u)$. Therefore $\left(u^{T},(S(u))^{T}\right)$ solves the following coupling system

$$
\left\{\begin{array}{l}
u_{t}=K(u) \\
v_{t}=K^{\prime}(u)[v]
\end{array}\right.
$$

if $S \in T(M)$ is found to be a symmetry of $u_{t}=K(u)$. This system (2.8) has been carefully considered upon introducing the perturbation bundle [1]. It is the firstorder standard perturbation system of $u_{t}=K(u)$, introduced in Ref. [5]. Since it keeps complete integrability, it provides us with an integrable coupling of the original system $u_{t}=K(u)$. Therefore the symmetry problem is viewed as a sub-case of general integrable couplings.

The commutator of the vector fields of the form $(K(u), A(u) v)^{T}$ with $A(u)$ being linear has a nice structure:

$$
\left[\left(\begin{array}{c}
K(u) \\
A(u) v
\end{array}\right),\left(\begin{array}{c}
S(u) \\
B(u) v
\end{array}\right)\right]=\left(\begin{array}{c}
{[K(u), S(u)]} \\
\llbracket A(u), B(u) \rrbracket v
\end{array}\right),
$$

where the commutator $[K(u), S(u)]$ is given by $(1.3)$ and the commutator $\llbracket A(u), B(u) \rrbracket$ of two linear operators $A(u), B(u)$ is defined by

$$
\llbracket A(u), B(u) \rrbracket=A^{\prime}(u)[S(u)]-B^{\prime}(u)[K(u)]+A(u) B(u)-B(u) A(u),
$$


which was used to analyze algebraic structures of Lax operators in [16]. Moreover for linearized operators, we can have

$$
\llbracket K^{\prime}(u), S^{\prime}(u) \rrbracket=T^{\prime}(u), T=[K, S], K, S \in T(M),
$$

which will be shown later on.

2.3. Candidates for integrable couplings. Let us illustrate the idea of how to construct candidates for integrable couplings by perturbations. Assume that an unperturbed system is given by

$$
u_{t}=K(u), K \in T(M),
$$

and we want to construct its integrable couplings. To this end, let us choose a simple perturbed system

$$
u_{t}=K(u)+\varepsilon K(u),
$$

which is analytic with respect to $\varepsilon$ of course, as an initial system. Obviously this system doesn't change integrable properties of the original system (2.10). In practice, we can have lots of choices of such perturbed systems. For example, if the system (2.10) has a symmetry $S \in T(M)$, then we can choose either $u_{t}=K(u)+\varepsilon S(u)$ or $u_{t}=K(u)+\varepsilon^{2} S(u)$ as another initial perturbed system. According to the definition of the perturbation systems in (2.7), the first-order perturbation system of the above perturbed system (2.11) reads as

$$
\left\{\begin{array}{l}
\eta_{0 t}=K\left(\eta_{0}\right) \\
\eta_{1 t}=K^{\prime}\left(\eta_{0}\right)\left[\eta_{1}\right]+K\left(\eta_{0}\right)
\end{array}\right.
$$

This coupling system is a candidate that we want to construct for getting integrable couplings of the original system $u_{t}=K(u)$. In fact, we will verify that the perturbation defined by (2.1) preserves complete integrability. Therefore the above coupling system (2.12) is an integrable coupling of the original system $u_{t}=K(u)$, provided that $u_{t}=K(u)$ itself is integrable. The realization of more integrable couplings, such as local $2+1$ dimensional bi-Hamiltonian systems, can be found from an application to the KdV hierarchy in the next section.

2.4. Structures of perturbation operators. Rather than working with concrete examples, we would like to establish general structures for three kinds of perturbation operators. The following three theorems will show us how to construct them explicitly. For the proof of the theorems, we first need to prove a basic result about the Gateaux derivative of the perturbation tensor fields.

LemMA 2.1. Let $X=X(\varepsilon) \in T_{s}^{r}(M)$ be analytic with respect to $\varepsilon$ and assume that the vector field $\bar{S}_{N}=\left(S_{0}^{T}, S_{1}^{T}, \cdots, S_{N}^{T}\right)^{T} \in T\left(\hat{M}_{N}\right)$, where all sub-vectors $S_{i}, 0 \leq$ $i \leq N$, are of the same dimension. Then the following equalities hold:

$$
\left(\left.\frac{\partial^{i} X\left(\hat{u}_{N}, \varepsilon\right)}{\partial \varepsilon^{i}}\right|_{\varepsilon=0}\right)^{\prime}\left(\hat{\eta}_{N}\right)\left[\bar{S}_{N}\right]=\left.\frac{\partial^{i}}{\partial \varepsilon^{i}}\right|_{\varepsilon=0} X^{\prime}\left(\hat{u}_{N}, \varepsilon\right)\left[\sum_{j=0}^{N} \varepsilon^{j} S_{j}\right], 0 \leq i \leq N .
$$

REMARK. Note that in (2.13), we have adopted the notation

$$
X^{\prime}\left(\hat{u}_{N}, \varepsilon\right)[K(u)]=\left(X\left(\hat{u}_{N}, \varepsilon\right)\right)^{\prime}\left(\hat{u}_{N}\right)[K(u)], X=X(\varepsilon) \in T_{s}^{r}(M), K \in T(M),
$$


in order to save space. The same notation will be used in the remainder of the paper.

Proof. Let us first observe the Taylor series

$$
X\left(\hat{u}_{N}, \varepsilon\right)=\left.\sum_{i=0}^{N} \frac{\varepsilon^{i}}{i !} \frac{\partial^{i} X\left(\hat{u}_{N}, \varepsilon\right)}{\partial \varepsilon^{i}}\right|_{\varepsilon=0}+\mathrm{o}\left(\varepsilon^{N}\right)
$$

It follows that

$$
\left(X\left(\hat{u}_{N}, \varepsilon\right)\right)^{\prime}\left(\hat{\eta}_{N}\right)\left[\bar{S}_{N}\right]=\sum_{i=0}^{N} \frac{\varepsilon^{i}}{i !}\left(\left.\frac{\partial^{i} X\left(\hat{u}_{N}, \varepsilon\right)}{\partial \varepsilon^{i}}\right|_{\varepsilon=0}\right)^{\prime}\left(\hat{\eta}_{N}\right)\left[\bar{S}_{N}\right]+\mathrm{o}\left(\varepsilon^{N}\right) .
$$

Secondly, we can compute that

$$
\left(X\left(\hat{u}_{N}, \varepsilon\right)\right)^{\prime}\left(\hat{\eta}_{N}\right)\left[\bar{S}_{N}\right]=\left.\frac{\partial}{\partial \delta}\right|_{\delta=0} X\left(\hat{u}_{N}+\delta \sum_{j=0}^{N} \varepsilon^{j} S_{j}, \varepsilon\right)=X^{\prime}\left(\hat{u}_{N}, \varepsilon\right)\left[\sum_{j=0}^{N} \varepsilon^{j} S_{j}\right] .
$$

A combination of the above two equalities leads to the required equalities in (2.13), again according to the Taylor series. The proof is completed.

THEOREM 2.2. If the operator $\Phi=\Phi(\varepsilon): T(M) \rightarrow T(M)$ being analytic with respect to $\varepsilon$ is hereditary, then the following operator $\hat{\Phi}_{N}: T\left(\hat{M}_{N}\right) \rightarrow T\left(\hat{M}_{N}\right)$ defined by

$$
\begin{aligned}
& \left(\operatorname{per}_{N} \Phi\right)\left(\hat{\eta}_{N}\right)=\hat{\Phi}_{N}\left(\hat{\eta}_{N}\right) \\
& =\left[\left(\hat{\Phi}_{N}\left(\hat{\eta}_{N}\right)\right)_{i j}\right]_{i, j=0,1, \cdots, N}=\left[\left.\frac{1}{(i-j) !} \frac{\partial^{i-j} \Phi\left(\hat{u}_{N}, \varepsilon\right)}{\partial \varepsilon^{i-j}}\right|_{\varepsilon=0}\right]_{q(N+1) \times q(N+1)}
\end{aligned}
$$

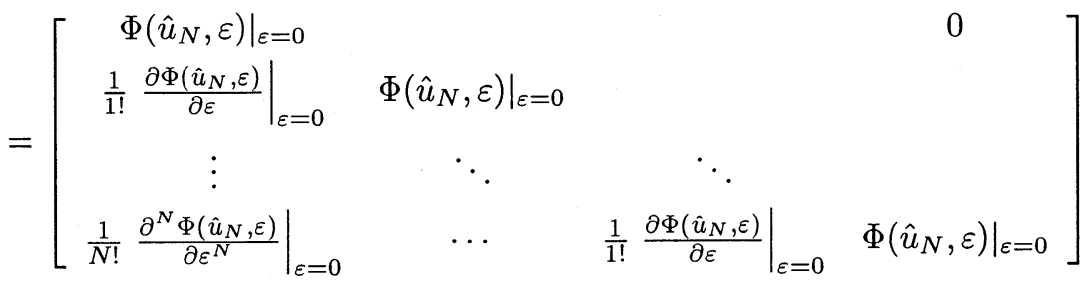

is also hereditary, where $\hat{u}_{N}$ is a perturbation series defined by (2.1).

Proof. Let $\bar{K}_{N}=\left(K_{0}^{T}, K_{1}^{T}, \cdots, K_{N}^{T}\right)^{T}, \bar{S}_{N}=\left(S_{0}^{T}, S_{1}^{T}, \cdots, S_{N}^{T}\right)^{T} \in T\left(\hat{M}_{N}\right)$, where the sub-vectors $K_{i}, S_{i}, 0 \leq i \leq N$, are of the same dimension. Since $\hat{\Phi}_{N}\left(\hat{\eta}_{N}\right)$ is obviously linear, we only need to prove that

$$
\begin{aligned}
& \hat{\Phi}_{N}^{\prime}\left(\hat{\eta}_{N}\right)\left[\hat{\Phi}_{N}\left(\hat{\eta}_{N}\right) \bar{K}_{N}\right] \bar{S}_{N}-\hat{\Phi}_{N}\left(\hat{\eta}_{N}\right) \hat{\Phi}_{N}^{\prime}\left(\hat{\eta}_{N}\right)\left[\bar{K}_{N}\right] \bar{S}_{N} \\
& -\hat{\Phi}_{N}^{\prime}\left(\hat{\eta}_{N}\right)\left[\hat{\Phi}_{N}\left(\hat{\eta}_{N}\right) \bar{S}_{N}\right] \bar{K}_{N}+\hat{\Phi}_{N}\left(\hat{\eta}_{N}\right) \hat{\Phi}_{N}^{\prime}\left(\hat{\eta}_{N}\right)\left[\bar{S}_{N}\right] \bar{K}_{N}=0
\end{aligned}
$$

according to Definition 1.4. In what follows, we are going to prove this equality.

First, we immediately obtain the $i$-th element of the vector field $\hat{\Phi}_{N}\left(\hat{\eta}_{N}\right) \bar{K}_{N}$ and the element in the $(i, j)$ position of the matrix $\hat{\Phi}_{N}^{\prime}\left(\hat{\eta}_{N}\right)\left[\bar{K}_{N}\right]$ :

$$
\left(\hat{\Phi}_{N}\left(\hat{\eta}_{N}\right) \bar{K}_{N}\right)_{i}=\left.\sum_{j=0}^{i} \frac{1}{(i-j) !} \frac{\partial^{i-j} \Phi\left(\hat{u}_{N}, \varepsilon\right)}{\partial \varepsilon^{i-j}}\right|_{\varepsilon=0} K_{j}, 0 \leq i \leq N
$$




$$
\begin{aligned}
& \left(\hat{\Phi}_{N}^{\prime}\left(\hat{\eta}_{N}\right)\left[\bar{K}_{N}\right]\right)_{i j}=\frac{1}{(i-j) !}\left(\left.\frac{\partial^{i-j} \Phi\left(\hat{u}_{N}, \varepsilon\right)}{\partial \varepsilon^{i-j}}\right|_{\varepsilon=0}\right)^{\prime}\left(\hat{\eta}_{N}\right)\left[\bar{K}_{N}\right] \\
& =\left.\frac{1}{(i-j) !} \frac{\partial^{i-j}}{\partial \varepsilon^{i-j}}\right|_{\varepsilon=0}\left(\Phi^{\prime}\left(\hat{u}_{N}, \varepsilon\right)\left[\sum_{k=0}^{N} \varepsilon^{k} K_{k}\right]\right), 0 \leq i, j \leq N,
\end{aligned}
$$

the last equality of which follows from Lemma 2.1 .

Now we can compute the $i$-th element of $\hat{\Phi}_{N}^{\prime}\left(\hat{\eta}_{N}\right)\left[\hat{\Phi}_{N}\left(\hat{\eta}_{N}\right) \bar{K}_{N}\right] \bar{S}_{N}$ as follows:

$$
\begin{aligned}
& \left(\hat{\Phi}_{N}^{\prime}\left(\hat{\eta}_{N}\right)\left[\hat{\Phi}_{N}\left(\hat{\eta}_{N}\right) \bar{K}_{N}\right] \bar{S}_{N}\right)_{i} \\
= & \left.\sum_{j=0}^{i} \frac{1}{(i-j) !} \frac{\partial^{i-j}}{\partial \varepsilon^{i-j}}\right|_{\varepsilon=0} \Phi^{\prime}\left(\hat{u}_{N}, \varepsilon\right)\left[\left.\sum_{k=0}^{N} \varepsilon^{k} \sum_{l=0}^{k} \frac{1}{(k-l) !} \frac{\partial^{k-l} \Phi\left(\hat{u}_{N}, \varepsilon\right)}{\partial \varepsilon^{k-l}}\right|_{\varepsilon=0} K_{l}\right] S_{j} \\
= & \left.\sum_{j=0}^{i} \frac{1}{(i-j) !} \frac{\partial^{i-j}}{\partial \varepsilon^{i-j}}\right|_{\varepsilon=0} \Phi^{\prime}\left(\hat{u}_{N}, \varepsilon\right)\left[\left.\sum_{l=0}^{N} \varepsilon^{l} \sum_{k=l}^{N} \frac{\varepsilon^{k-l}}{(k-l) !} \frac{\partial^{k-l} \Phi\left(\hat{u}_{N}, \varepsilon\right)}{\partial \varepsilon^{k-l}}\right|_{\varepsilon=0} K_{l}\right] S_{j} \\
= & \left.\sum_{j=0}^{i} \frac{1}{(i-j) !} \frac{\partial^{i-j}}{\partial \varepsilon^{i-j}}\right|_{\varepsilon=0} \Phi^{\prime}\left(\hat{u}_{N}, \varepsilon\right)\left[\sum_{l=0}^{N} \varepsilon^{l}\left(\Phi\left(\hat{u}_{N}, \varepsilon\right)+o\left(\varepsilon^{N-l}\right)\right) K_{l}\right] S_{j} \\
= & \left.\sum_{j=0}^{i} \frac{1}{(i-j) !} \frac{\partial^{i-j}}{\partial \varepsilon^{i-j}}\right|_{\varepsilon=0} \Phi^{\prime}\left(\hat{u}_{N}, \varepsilon\right)\left[\sum_{l=0}^{N} \varepsilon^{l} \Phi\left(\hat{u}_{N}, \varepsilon\right) K_{l}\right] S_{j} \\
= & \left.\sum_{0 \leq j+l \leq i} \frac{1}{(i-j-l) !} \frac{\partial^{i-j-l}}{\partial \varepsilon^{i-j-l}}\right|_{\varepsilon=0} \Phi^{\prime}\left(\hat{u}_{N}, \varepsilon\right)\left[\Phi\left(\hat{u}_{N}, \varepsilon\right) K_{l}\right] S_{j}, 0 \leq i \leq N .
\end{aligned}
$$

On the other hand, we can compute the $i$-th element of $\hat{\Phi}_{N}\left(\hat{\eta}_{N}\right) \hat{\Phi}_{N}^{\prime}\left(\hat{\eta}_{N}\right)\left[\bar{K}_{N}\right] \bar{S}_{N}$ as follows:

$$
\begin{aligned}
& \left(\hat{\Phi}_{N}\left(\hat{\eta}_{N}\right) \hat{\Phi}_{N}^{\prime}\left(\hat{\eta}_{N}\right)\left[\bar{K}_{N}\right] \bar{S}_{N}\right)_{i} \\
= & \left.\left.\sum_{j=0}^{i} \frac{1}{(i-j) !} \frac{\partial^{i-j} \Phi\left(\hat{u}_{N}, \varepsilon\right)}{\partial \varepsilon^{i-j}}\right|_{\varepsilon=0} \sum_{k=0}^{j} \frac{1}{(j-k) !} \frac{\partial^{j-k}}{\partial \varepsilon^{j-k}}\right|_{\varepsilon=0} \Phi^{\prime}\left(\hat{u}_{N}, \varepsilon\right)\left[\sum_{l=0}^{N} \varepsilon^{l} K_{l}\right] S_{k} \\
= & \left.\left.\sum_{j=0}^{i} \frac{1}{(i-j) !} \frac{\partial^{i-j} \Phi\left(\hat{u}_{N}, \varepsilon\right)}{\partial \varepsilon^{i-j}}\right|_{\varepsilon=0} \sum_{k=0}^{j} \sum_{l=0}^{j-k} \frac{1}{(j-k-l) !} \frac{\partial^{j-k-l}}{\partial \varepsilon^{j-k-l}}\right|_{\varepsilon=0} \Phi^{\prime}\left(\hat{u}_{N}, \varepsilon\right)\left[K_{l}\right] S_{k} \\
= & \left.\left.\sum_{k=0}^{i} \sum_{j=k}^{i} \sum_{l=0}^{j-k} \frac{1}{(i-j) !(j-k-l) !} \frac{\partial^{i-j} \Phi\left(\hat{u}_{N}, \varepsilon\right)}{\partial \varepsilon^{i-j}}\right|_{\varepsilon=0} \frac{\partial^{j-k-l}}{\partial \varepsilon^{j-k-l}}\right|_{\varepsilon=0} \Phi^{\prime}\left(\hat{u}_{N}, \varepsilon\right)\left[K_{l}\right] S_{k} \\
= & \left.\left.\sum_{k=0}^{i} \sum_{l=0}^{i-k} \sum_{j=k+l}^{i} \frac{\partial^{i-j} \Phi\left(\hat{u}_{N}, \varepsilon\right)}{\partial \varepsilon^{i-j}}\right|_{\varepsilon=0} \frac{\partial^{j-k-l}}{\partial \varepsilon^{j-k-l}}\right|_{\varepsilon=0} \Phi^{\prime}\left(\hat{u}_{N}, \varepsilon\right)\left[K_{l}\right] S_{k} \\
= & \left.\sum_{k=0}^{i} \sum_{l=0}^{i-k} \frac{1}{(i-k-l) !} \frac{\partial^{i-k-l}}{\partial \varepsilon^{i-k-l}}\right|_{\varepsilon=0}\left(\Phi\left(\hat{u}_{N}, \varepsilon\right) \Phi^{\prime}\left(\hat{u}_{N}, \varepsilon\right)\left[K_{l}\right] S_{k}\right) \\
= & \left.\sum_{0 \leq k+l \leq i} \frac{1}{(i-k-l) !} \frac{\partial^{i-k-l}}{\partial \varepsilon^{i-k-l}}\right|_{\varepsilon=0}\left(\Phi\left(\hat{u}_{N}, \varepsilon\right) \Phi^{\prime}\left(\hat{u}_{N}, \varepsilon\right)\left[K_{l}\right] S_{k}\right), 0 \leq i \leq N .
\end{aligned}
$$

Therefore, it follows from the hereditary property of $\Phi(u, \varepsilon)$ that each element in the left-hand side of (2.16) is equal to zero, which means that (2.16) is true. The proof is completed. $\square$ 
THEOREM 2.3. If the operator $J=J(\varepsilon): T^{*}(M) \rightarrow T(M)$ being analytic with respect to $\varepsilon$ is Hamiltonian, then the following operator $\hat{J}_{N}: T^{*}\left(\hat{M}_{N}\right) \rightarrow T\left(\hat{M}_{N}\right)$ defined by

$$
\begin{aligned}
& \left(\operatorname{per}_{N} J\right)\left(\hat{\eta}_{N}\right)=\hat{J}_{N}\left(\hat{\eta}_{N}\right)=\left[\left(\hat{J}_{N}\left(\hat{\eta}_{N}\right)\right)_{i j}\right]_{i, j=0,1, \cdots, N} \\
& =\left[\left.\frac{1}{(i+j-N) !} \frac{\partial^{i+j-N} J\left(\hat{u}_{N}, \varepsilon\right)}{\partial \varepsilon^{i+j-N}}\right|_{\varepsilon=0}\right]_{q(N+1) \times q(N+1)} \\
& =\left[\begin{array}{cccc}
0 & & & \left.J\left(\hat{u}_{N}, \varepsilon\right)\right|_{\varepsilon=0} \\
& & \left.J\left(\hat{u}_{N}, \varepsilon\right)\right|_{\varepsilon=0} & \left.\frac{1}{1 !} \frac{\partial J\left(\hat{u}_{N}, \varepsilon\right)}{\partial \varepsilon}\right|_{\varepsilon=0} \\
\left.J\left(\hat{u}_{N}, \varepsilon\right)\right|_{\varepsilon=0} & \left.\frac{1}{1 !} \frac{\partial J\left(\hat{u}_{N}, \varepsilon\right)}{\partial \varepsilon}\right|_{\varepsilon=0} & \therefore & \vdots \\
N ! & \cdots & \left.\frac{\partial^{N} J\left(\hat{u}_{N}, \varepsilon\right)}{\partial \varepsilon^{N}}\right|_{\varepsilon=0}
\end{array}\right]
\end{aligned}
$$

is also Hamiltonian, where $\hat{u}_{N}$ is a perturbation series defined by (2.1).

Proof. $\quad$ Let $\bar{\alpha}_{N}=\left(\alpha_{0}^{T}, \alpha_{1}^{T}, \cdots, \alpha_{N}^{T}\right)^{T}, \quad \bar{\beta}_{N}=\left(\beta_{0}^{T}, \beta_{1}^{T}, \cdots, \beta_{N}^{T}\right)^{T}, \quad \bar{\gamma}_{N}=$ $\left(\gamma_{0}^{T}, \gamma_{1}^{T}, \cdots, \gamma_{N}^{T}\right)^{T} \in T^{*}\left(\hat{M}_{N}\right)$, where the sub-vectors $\alpha_{i}, \beta_{i}, \gamma_{i}, 0 \leq i \leq N$, are of the same dimension. It suffices to prove that

$$
<\bar{\alpha}_{N}, \hat{J}_{N}^{\prime}\left(\hat{\eta}_{N}\right)\left[\hat{J}_{N} \bar{\beta}_{N}\right] \bar{\gamma}_{N}>+\operatorname{cycle}\left(\bar{\alpha}_{N}, \bar{\beta}_{N}, \bar{\gamma}_{N}\right)=0
$$

since there is no problem on the linearity and the skew-symmetric property for the perturbation operator $\hat{J}_{N}\left(\hat{\eta}_{N}\right)$.

First, based on Lemma 2.1, we can calculate the element in the $(i, j)$ position of the matrix $\hat{J}_{N}^{\prime}\left(\hat{\eta}_{N}\right)\left[\hat{J}_{N}\left(\hat{\eta}_{N}\right) \bar{\beta}_{N}\right]$ as follows:

$$
\begin{aligned}
& \left(\hat{J}_{N}^{\prime}\left(\hat{\eta}_{N}\right)\left[\hat{J}_{N}\left(\hat{\eta}_{N}\right) \bar{\beta}_{N}\right]\right)_{i j} \\
= & \left.\frac{1}{(i+j-N) !} \frac{\partial^{i+j-N}}{\partial \varepsilon^{i+j-N}}\right|_{\varepsilon=0} J^{\prime}\left(\hat{u}_{N}, \varepsilon\right)\left[\sum_{l=0}^{N} \varepsilon^{l}\left(\hat{J}_{N} \bar{\beta}_{N}\right)_{l}\right] \\
= & \left.\frac{1}{(i+j-N) !} \frac{\partial^{i+j-N}}{\partial \varepsilon^{i+j-N}}\right|_{\varepsilon=0} \times \\
= & \left.\left.\frac{1}{(i+j-N) !} \frac{\partial^{\prime}\left(\hat{u}_{N}, \varepsilon\right)\left[\left.\sum_{l=0}^{N} \varepsilon^{l} \sum_{k=N-l}^{N} \frac{1}{\partial \varepsilon^{i+j-N}}\right|_{\varepsilon=0} \times\right.}{(k+l-N) !} \frac{\partial^{k+l-N} J\left(\hat{u}_{N}, \varepsilon\right)}{\partial \varepsilon^{k+l-N}}\right|_{\varepsilon=0} \beta_{k}\right] \\
= & \left.\left.\left.\frac{1}{(i+j-N) !} \frac{\partial^{\prime}\left(\hat{u}_{N}, \varepsilon\right)\left[\left.\sum_{k=0}^{N} \frac{\varepsilon^{N-k}}{\partial \varepsilon^{i+j-N}}\right|_{\varepsilon=0} ^{N} \frac{\varepsilon^{k+l-N}}{J^{\prime}\left(\hat{u}_{N}, \varepsilon\right)\left[\sum_{k=0}^{N} \varepsilon^{N-k}\left(J\left(\hat{u}_{N}, \varepsilon\right)+o\left(\varepsilon^{k}\right)\right) \beta_{k}\right]}\right.}{\left.\partial \varepsilon^{k+l-N}\right]}\right|_{\varepsilon=0}\right) \beta_{k}\right] \\
= & \left.\frac{1}{(i+j-N) !} \frac{\partial^{i+j-N}}{\partial \varepsilon^{i+j-N}}\right|_{\varepsilon=0} J^{\prime}\left(\hat{u}_{N}, \varepsilon\right)\left[\sum_{k=0}^{N} \varepsilon^{N-k} J\left(\hat{u}_{N}, \varepsilon\right) \beta_{k}\right] \\
= & \left.\sum_{k=0}^{N} \frac{1}{(i+j-N) !} \frac{\partial^{i+j-N}}{\partial \varepsilon^{i+j-N}}\right|_{\varepsilon=0}\left(\varepsilon^{N-k} J^{\prime}\left(\hat{u}_{N}, \varepsilon\right)\left[J\left(\hat{u}_{N}, \varepsilon\right) \beta_{k}\right]\right)
\end{aligned}
$$




$$
=\left.\sum_{k=2 N-(i+j)}^{N} \frac{1}{(i+j+k-2 N) !} \frac{\partial^{i+j+k-2 N}}{\partial \varepsilon^{i+j+k-2 N}}\right|_{\varepsilon=0} J^{\prime}\left(\hat{u}_{N}, \varepsilon\right)\left[J\left(\hat{u}_{N}, \varepsilon\right) \beta_{k}\right], 0 \leq i, j \leq N .
$$

In what follows, let us give the remaining proof for the case of

$$
\eta_{i}=\eta_{i}\left(y_{0}, y_{1}, t\right)=\eta_{i}(x, \varepsilon x, t), 0 \leq i \leq N .
$$

Suppose that the duality between cotangent vectors and tangent vectors is given by

$$
<\alpha, K>=\int_{\mathbb{R}^{p}} \alpha^{T} K d x, x \in \mathbb{R}^{p}, \alpha \in T^{*}(M), K \in T(M) .
$$

Let us consider the case of $x \in \mathbb{R}$ without loss of generality. For brevity, we set

$$
\begin{aligned}
& F_{i j k}\left(\bar{\alpha}_{N}, \bar{\beta}_{N}, \bar{\gamma}_{N}, \hat{\partial}_{x}\right) \\
& =\left(\alpha_{i}^{T} J^{\prime}\left(\hat{u}_{N}, \varepsilon\right)\left[J\left(\hat{u}_{N}, \varepsilon\right) \beta_{k}\right] \gamma_{j}+\operatorname{cycle}\left(\alpha_{i}, \beta_{k}, \gamma_{j}\right)\right), \quad 0 \leq i, j, k \leq N,
\end{aligned}
$$

where $\hat{\partial}_{x}=\partial_{y_{0}}+\varepsilon \partial_{y_{1}}$, owing to (2.19), and we assume that the original Hamiltonian operator $J(u, \varepsilon)$ involves the differential operator $\partial_{x}$. Then we can have

$$
\begin{aligned}
& <\bar{\alpha}_{N}, \hat{J}_{N}^{\prime}\left(\hat{\eta}_{N}\right)\left[\hat{J}_{N} \bar{\beta}_{N}\right] \bar{\gamma}_{N}>+\operatorname{cycle}\left(\bar{\alpha}_{N}, \bar{\beta}_{N}, \bar{\gamma}_{N}\right) \\
= & \left.\int_{-\infty}^{\infty} \int_{-\infty}^{\infty} \sum_{-_{2 N} \leq i+j+k \leq 3 N} \frac{1}{(i+j+k-2 N) !} \frac{\partial^{i+j+k-2 N}}{\partial \varepsilon^{i+j+k-2 N}}\right|_{\varepsilon=0} F_{i j k}\left(\bar{\alpha}_{N}, \bar{\beta}_{N}, \bar{\gamma}_{N}, \hat{\partial}_{x}\right) d y_{0} d y_{1} .
\end{aligned}
$$

In order to apply the Jacobi identity of $J(u, \varepsilon)$, we make a dependent variable transformation

$$
y_{0}=p, y_{1}=q+\varepsilon p
$$

frc.n which it follows that

$$
\partial_{p}=\partial_{y_{0}}+\varepsilon \partial_{y_{1}}, \partial_{q}=\partial_{y_{1}} .
$$

Now we can continue to compute that

$$
\begin{aligned}
& <\bar{\alpha}_{N}, \hat{J}_{N}^{\prime}\left(\hat{\eta}_{N}\right)\left[\hat{J}_{N} \bar{\beta}_{N}\right] \bar{\gamma}_{N}>+\operatorname{cycle}\left(\bar{\alpha}_{N}, \bar{\beta}_{N}, \bar{\gamma}_{N}\right) \\
= & \left.\int_{-\infty}^{\infty} \int_{-\infty}^{\infty} \sum_{2 N \leq i+j+k \leq 3 N} \frac{1}{(i+j+k-2 N) !} \frac{\partial^{i+j+k-2 N}}{\partial \varepsilon^{i+j+k-2 N}}\right|_{\varepsilon=0} \times \\
& F_{i j k}\left(\bar{\alpha}_{N}, \bar{\beta}_{N}, \bar{\gamma}_{N}, \partial_{p}\right)\left|\operatorname{det}\left[\begin{array}{cc}
\frac{\partial y_{0}}{\partial p} & \frac{\partial y_{0}}{\partial q} \\
\frac{\partial y_{1}}{\partial p} & \frac{\partial y_{1}}{\partial q}
\end{array}\right]\right| d p d q \\
= & \int_{-\infty}^{\infty}\left(\left.\sum_{2 N \leq i+j+k \leq 3 N} \frac{1}{(i+j+k-2 N) !} \frac{\partial^{i+j+k-2 N}}{\partial \varepsilon^{i+j+k-2 N}}\right|_{\varepsilon=0} \times\right. \\
= & \int_{-\infty}^{\infty} 0 d q=0 .
\end{aligned}
$$

In the last but one step, we have utilized the Jacobi identity of $J(u, \varepsilon)$. 
The method used here for showing the Jacobi identity can be extended to the other cases of the perturbations. Therefore the required result is proved.

Similarly, we can show the following structure for the perturbation symplectic operators.

THEOREM 2.4. If the operator $\Theta=\Theta(\varepsilon): T(M) \rightarrow T^{*}(M)$ being analytic with respect to $\varepsilon$ is symplectic, then the following operator $\hat{\Theta}_{N}: T\left(\hat{M}_{N}\right) \rightarrow T^{*}\left(\hat{M}_{N}\right)$ defined by

$$
\begin{aligned}
& \left(\operatorname{per}_{N} \Theta\right)\left(\hat{\eta}_{N}\right)=\hat{\Theta}_{N}\left(\hat{\eta}_{N}\right)=\left[\left(\hat{\Theta}_{N}\left(\hat{\eta}_{N}\right)\right)_{i j}\right]_{i, j=0,1, \cdots, N} \\
& =\left[\left.\frac{1}{(N-i-j) !} \frac{\partial^{N-i-j} \Theta\left(\hat{u}_{N}, \varepsilon\right)}{\partial \varepsilon^{N-i-j}}\right|_{\varepsilon=0}\right]_{q(N+1) \times q(N+1)} \\
& =\left[\begin{array}{cccc}
\left.\frac{1}{N !} \frac{\partial^{N} \Theta\left(\hat{u}_{N}, \varepsilon\right)}{\partial \varepsilon^{N}}\right|_{\varepsilon=0} & \ldots & \left.\frac{1}{1 !} \frac{\partial \Theta\left(\hat{u}_{N}, \varepsilon\right)}{\partial \varepsilon}\right|_{\varepsilon=0} & \left.\Theta\left(\hat{u}_{N}, \varepsilon\right)\right|_{\varepsilon=0} \\
\vdots & \therefore & \therefore & \\
\left.\frac{1}{1 !} \frac{\partial^{N} \Theta\left(\hat{u}_{N}, \varepsilon\right)}{\partial \varepsilon^{N}}\right|_{\varepsilon=0} & \left.\Theta\left(\hat{u}_{N}, \varepsilon\right)\right|_{\varepsilon=0} & & \\
\left.\Theta\left(\hat{u}_{N}, \varepsilon\right)\right|_{\varepsilon=0} & & & 0
\end{array}\right]
\end{aligned}
$$

is also symplectic, where $\hat{u}_{N}$ is a perturbation series defined by (2.1).

2.5. Integrable Properties. In this sub-section, we study integrable properties of the perturbation systems defined by (2.7), which include recursion hereditary operators, $K$-symmetries (i.e., time independent symmetries), master-symmetries, Lax representations and zero curvature representations, Hamiltonian formulations and etc. Simultaneously we establish explicit structures for constructing other perturbation objects such as spectral problems, Hamiltonian functionals, and cotangent vector fields.

THEOREM 2.5. Let $K=K(\varepsilon) \in T(M)$ be analytic with respect to $\varepsilon$ and assume that $\Phi=\Phi(\varepsilon): T(M) \rightarrow T(M)$ is a recursion operator of $u_{t}=K(u, \varepsilon)$. Then the operator $\hat{\Phi}_{N}: T\left(\hat{M}_{N}\right) \rightarrow T\left(\hat{M}_{N}\right)$ determined by (2.15) is a recursion operator of the perturbation system $\hat{\eta}_{N t}=\hat{K}_{N}\left(\hat{\eta}_{N}\right)$ defined by (2.7). Therefore if $u_{t}=K(u, \varepsilon)$ has a hereditary recursion operator $\Phi(u, \varepsilon)$, then the perturbation system $\hat{\eta}_{N t}=\hat{K}_{N}\left(\hat{\eta}_{N}\right)$ has a hereditary recursion operator $\hat{\Phi}_{N}\left(\hat{\eta}_{N}\right)$.

Proof. Let $\bar{S}_{N}=\left(S_{0}^{T}, S_{1}^{T}, \cdots, S_{N}^{T}\right)^{T} \in T\left(\hat{M}_{N}\right)$, where the sub-vectors $S_{i}, 0 \leq$ $i \leq N$, are of the same dimension. By Lemma 2.1, we can compute that

$$
\begin{aligned}
& \left(\left.\frac{\partial^{k} \Phi\left(\hat{u}_{N}, \varepsilon\right)}{\partial \varepsilon^{k}}\right|_{\varepsilon=0}\right)^{\prime}\left(\hat{\eta}_{N}\right)\left[\hat{K}_{N}\right]=\left.\frac{\partial^{k}}{\partial \varepsilon^{k}}\right|_{\varepsilon=0} \Phi^{\prime}\left(\hat{u}_{N}, \varepsilon\right)\left[\sum_{j=0}^{N} \varepsilon^{j} K^{(j)}\right] \\
= & \left.\frac{\partial^{k}}{\partial \varepsilon^{k}}\right|_{\varepsilon=0} \Phi^{\prime}\left(\hat{u}_{N}, \varepsilon\right)\left[K\left(\hat{u}_{N}, \varepsilon\right)+o\left(\varepsilon^{N}\right)\right]=\left.\frac{\partial^{k} \Phi^{\prime}\left(\hat{u}_{N}, \varepsilon\right)\left[K\left(\hat{u}_{N}, \varepsilon\right)\right]}{\partial \varepsilon^{k}}\right|_{\varepsilon=0}, 0 \leq k \leq N,
\end{aligned}
$$

and

$$
\begin{aligned}
& \left(K^{(i)}\right)^{\prime}\left(\hat{\eta}_{N}\right)\left[\bar{S}_{N}\right]=\left.\frac{1}{i !} \frac{\partial^{i}}{\partial \varepsilon^{i}}\right|_{\varepsilon=0} K^{\prime}\left(\hat{u}_{N}, \varepsilon\right)\left[\sum_{k=0}^{N} \varepsilon^{k} S_{k}\right] \\
= & \left.\sum_{j=0}^{i} \frac{1}{(i-j) !} \frac{\partial^{i-j} K^{\prime}\left(\hat{u}_{N}, \varepsilon\right)\left[S_{j}\right]}{\partial \varepsilon^{i-j}}\right|_{\varepsilon=0}, 0 \leq i \leq N .
\end{aligned}
$$


Therefore, immediately from the first equality above, we obtain the $i$-th element of $\hat{\Phi}_{N}^{\prime}\left(\hat{\eta}_{N}\right)\left[\hat{K}_{N}\right] \bar{S}_{N}$ as follows:

$$
\left(\hat{\Phi}_{N}^{\prime}\left(\hat{\eta}_{N}\right)\left[\hat{K}_{N}\right] \bar{S}_{N}\right)_{i}=\left.\sum_{j=0}^{i} \frac{1}{(i-j) !} \frac{\partial^{i-j}\left(\Phi^{\prime}\left(\hat{u}_{N}, \varepsilon\right)\left[K\left(\hat{u}_{N}, \varepsilon\right)\right] S_{j}\right)}{\partial \varepsilon^{i-j}}\right|_{\varepsilon=0}, 0 \leq i \leq N .
$$

Based on the second equality above, we can make the following computation:

$$
\begin{aligned}
& \left(\hat{K}_{N}^{\prime}\left(\hat{\eta}_{N}\right)\left[\hat{\Phi}_{N}\left(\hat{\eta}_{N}\right) \bar{S}_{N}\right]\right)_{i} \\
= & \left.\sum_{k=0}^{i} \frac{1}{(i-k) !} \frac{\partial^{i-k}}{\partial \varepsilon^{i-k}}\right|_{\varepsilon=0}\left(K^{\prime}\left(\hat{u}_{N}, \varepsilon\right)\left[\left(\hat{\Phi}_{N}\left(\hat{\eta}_{N}\right) \bar{S}_{N}\right)_{k}\right]\right) \\
= & \left.\sum_{k=0}^{i} \frac{1}{(i-k) !} \frac{\partial^{i-k}}{\partial \varepsilon^{i-k}}\right|_{\varepsilon=0} K^{\prime}\left(\hat{u}_{N}, \varepsilon\right)\left[\left.\sum_{j=0}^{k} \frac{1}{(k-j) !} \frac{\partial^{k-j} \Phi\left(\hat{u}_{N}, \varepsilon\right)}{\partial \varepsilon^{k-j}}\right|_{\varepsilon=0} S_{j}\right] \\
= & \left.\sum_{j=0}^{i} \sum_{k=j}^{i} \frac{1}{(i-k) !(k-j) !} \frac{\partial^{i-k}}{\partial \varepsilon^{i-k}}\right|_{\varepsilon=0} K^{\prime}\left(\hat{u}_{N}, \varepsilon\right)\left[\left.\frac{\partial^{k-j} \Phi\left(\hat{u}_{N}, \varepsilon\right)}{\partial \varepsilon^{k-j}}\right|_{\varepsilon=0} S_{j}\right] \\
= & \left.\sum_{j=0}^{i} \sum_{k=j}^{i} \frac{1}{(i-j) !} \frac{\partial^{i-j}}{\partial \varepsilon^{i-j}}\right|_{\varepsilon=0}\left(\frac{\varepsilon^{k-j}}{(k-j) !} K^{\prime}\left(\hat{u}_{N}, \varepsilon\right)\left[\left.\frac{\partial^{k-j} \Phi\left(\hat{u}_{N}, \varepsilon\right)}{\partial \varepsilon^{k-j}}\right|_{\varepsilon=0} S_{j}\right]\right) \\
= & \left.\sum_{j=0}^{i} \frac{1}{(i-j) !} \frac{\partial^{i-j}}{\partial \varepsilon^{i-j}}\right|_{\varepsilon=0} K^{\prime}\left(\hat{u}_{N}, \varepsilon\right)\left[\left.\sum_{k=j}^{i} \frac{\varepsilon^{k-j}}{(k-j) !} \frac{\partial^{k-j} \Phi\left(\hat{u}_{N}, \varepsilon\right)}{\partial \varepsilon^{k-j}}\right|_{\varepsilon=0} S_{j}\right] \\
= & \left.\sum_{j=0}^{i} \frac{1}{(i-j) !} \frac{\partial^{i-j}}{\partial \varepsilon^{i-j}}\right|_{\varepsilon=0} K^{\prime}\left(\hat{u}_{N}, \varepsilon\right)\left[\Phi\left(\hat{u}_{N}\right) S_{j}+\mathrm{o}\left(\varepsilon^{i-j}\right)\right] \\
= & \sum_{j=0}^{i} \frac{1}{(i-j) !} \frac{\partial^{i-j}\left(K^{\prime}\left(\hat{u}_{N}, \varepsilon\right)\left[\Phi\left(\hat{u}_{N}, \varepsilon\right) S_{j}\right]\right)}{\partial \varepsilon^{i-j}} \quad, 0 \leq i \leq N ;
\end{aligned}
$$

$$
\begin{aligned}
& \left(\hat{\Phi}_{N}\left(\hat{\eta}_{N}\right) \hat{K}_{N}^{\prime}\left(\hat{\eta}_{N}\right)\left[\bar{S}_{N}\right]\right)_{i} \\
= & \left.\left.\sum_{k=0}^{i} \frac{1}{(i-k) !} \frac{\partial^{i-k} \Phi\left(\hat{u}_{N}, \varepsilon\right)}{\partial \varepsilon^{i-k}}\right|_{\varepsilon=0} \sum_{j=0}^{k} \frac{1}{(k-j) !} \frac{\partial^{k-j} K^{\prime}\left(\hat{u}_{N}, \varepsilon\right)\left[S_{j}\right]}{\partial \varepsilon^{k-j}}\right|_{\varepsilon=0} \\
= & \left.\left.\sum_{j=0}^{i} \sum_{k=j}^{i} \frac{1}{(i-k) !(k-j) !} \frac{\partial^{i-k} \Phi\left(\hat{u}_{N}, \varepsilon\right)}{\partial \varepsilon^{i-k}}\right|_{\varepsilon=0} \frac{\partial^{k-j} K^{\prime}\left(\hat{u}_{N}, \varepsilon\right)\left[S_{j}\right]}{\partial \varepsilon^{k-j}}\right|_{\varepsilon=0} \\
= & \left.\sum_{j=0}^{i} \frac{1}{(i-j) !} \frac{\partial^{i-j}\left(\Phi\left(\hat{u}_{N}, \varepsilon\right) K^{\prime}\left(\hat{u}_{N}, \varepsilon\right)\left[S_{j}\right]\right)}{\partial \varepsilon^{i-k}}\right|_{\varepsilon=0}, 0 \leq i \leq N .
\end{aligned}
$$

It follows directly from the above three equalities above (2.25), (2.26) and (2.27) that

$$
\begin{aligned}
& \frac{\partial \hat{\Phi}_{N}}{\partial t}\left(\hat{\eta}_{N}\right) \bar{S}_{N}+\hat{\Phi}_{N}^{\prime}\left(\hat{\eta}_{N}\right)\left[\hat{K}_{N}\left(\hat{\eta}_{N}\right)\right] \bar{S}_{N}-\hat{K}_{N}^{\prime}\left(\hat{\eta}_{N}\right)\left[\hat{\Phi}_{N}\left(\hat{\eta}_{N}\right) \bar{S}_{N}\right]+\hat{\Phi}_{N}\left(\hat{\eta}_{N}\right) \hat{K}_{N}^{\prime}\left(\hat{\eta}_{N}\right)\left[\bar{S}_{N}\right] \\
& =0 .
\end{aligned}
$$


According to Definition 1.3, this implies that the perturbation operator $\hat{\Phi}_{N}\left(\hat{\eta}_{N}\right)$ defined by $(2.15)$ is a recursion operator of $\hat{\eta}_{N t}=\hat{K}_{N}\left(\hat{\eta}_{N}\right)$. A combination with Theorem 2.2 gives rise to the proof of the second required conclusion. The proof is finished.

THEOREM 2.6. Let $K=K(\varepsilon), S=S(\varepsilon) \in T(M)$ be analytic with respect to $\varepsilon$. For two perturbation vector fields $\hat{K}_{N}, \hat{S}_{N} \in T\left(\hat{M}_{N}\right)$ defined by (2.3), there exists the following relation:

$$
\left[\hat{K}_{N}\left(\hat{\eta}_{N}\right), \hat{S}_{N}\left(\hat{\eta}_{N}\right)\right]=\left(\hat{K}_{N}\right)^{\prime}\left(\hat{\eta}_{N}\right)\left[\hat{S}_{N}\left(\hat{\eta}_{N}\right)\right]-\left(\hat{S}_{N}\right)^{\prime}\left(\hat{\eta}_{N}\right)\left[\hat{K}_{N}\left(\hat{\eta}_{N}\right)\right]=\hat{T}_{N}\left(\hat{\eta}_{N}\right)
$$

where $\hat{T}_{N} \in T\left(\hat{M}_{N}\right)$ is the perturbation vector field of the vector field $T(\varepsilon)=[K(\varepsilon), S(\varepsilon)]$, defined by (2.3). Furthermore we can have the following:

(1) if $\sigma=\sigma(\varepsilon) \in T(M)$ is an $n$-th order master-symmetry of the perturbed system $u_{t}=K(u, \varepsilon)$, then $\hat{\sigma}_{N} \in T\left(\hat{M}_{N}\right)$ defined by (2.3) is an $n$-th order master-symmetry of the perturbation system $\hat{\eta}_{N t}=\hat{K}_{N}\left(\hat{\eta}_{N}\right)$ defined by (2.7);

(2) the perturbation system $\hat{\eta}_{N t}=\hat{K}_{N}\left(\hat{\eta}_{N}\right)$ defined by (2.7) possesses the same structure of symmetry algebras as the original perturbed system $u_{t}=K(u, \varepsilon)$.

Proof. As usual, assume that

$$
\hat{S}_{i}=\left(S^{(0) T}, S^{(1) T}, \cdots, S^{(i) T}\right)^{T}, \hat{\eta}_{i}=\left(\eta_{0}^{T}, \eta_{1}^{T}, \cdots, \eta_{i}^{T}\right)^{T}, \hat{u}_{i}=\sum_{k=0}^{i} \varepsilon^{k} \eta_{k}, 0 \leq i \leq N
$$

By the definition of the Gateaux derivative, we first have

$$
\begin{aligned}
& \left(K\left(\hat{u}_{i}, \varepsilon\right)\right)^{\prime}\left(\hat{\eta}_{i}\right)\left[\hat{S}_{i}\right]=\left.\frac{\partial}{\partial \delta}\right|_{\delta=0} K\left(\hat{u}_{i}+\delta \sum_{k=0}^{i} \varepsilon^{k} S^{(k)}, \varepsilon\right) \\
= & \left.\frac{\partial}{\partial \delta}\right|_{\delta=0} K\left(\hat{u}_{i}+\delta S\left(\hat{u}_{i}, \varepsilon\right)+\delta \mathrm{o}\left(\varepsilon^{i}\right), \varepsilon\right) \\
= & K^{\prime}\left(\hat{u}_{i}, \varepsilon\right)\left[S\left(\hat{u}_{i}, \varepsilon\right)\right]+\mathrm{o}\left(\varepsilon^{i}\right), 0 \leq i \leq N .
\end{aligned}
$$

Let us apply the equality above to the following Taylor series

$$
K\left(\hat{u}_{i}, \varepsilon\right)=\left.\sum_{k=0}^{i} \frac{\varepsilon^{k}}{k !} \frac{\partial^{k} K\left(\hat{u}_{i}, \varepsilon\right)}{\partial \varepsilon^{k}}\right|_{\varepsilon=0}+o\left(\varepsilon^{i}\right), 0 \leq i \leq N
$$

and then we arrive at

$$
K^{\prime}\left(\hat{u}_{i}, \varepsilon\right)\left[S\left(\hat{u}_{i}, \varepsilon\right)\right]=\sum_{k=0}^{i} \frac{\varepsilon^{k}}{k !}\left(\left.\frac{\partial^{k} K\left(\hat{u}_{i}, \varepsilon\right)}{\partial \varepsilon^{k}}\right|_{\varepsilon=0}\right)^{\prime}\left(\hat{\eta}_{i}\right)\left[\hat{S}_{i}\right]+\mathrm{o}\left(\varepsilon^{i}\right), 0 \leq i \leq N .
$$

Taking the $i$-th derivative with respect to $\varepsilon$ leads to

$$
\left(K^{\prime}(u, \varepsilon)[S(u, \varepsilon)]\right)^{(i)}\left(\hat{\eta}_{i}\right)=\left((K(u, \varepsilon))^{(i)}\right)^{\prime}\left(\hat{\eta}_{i}\right)\left[\hat{S}_{i}\right], 0 \leq i \leq N .
$$

Now it follows from (2.29) that for the $i$-th element of $\hat{T}_{N}$ we have

$$
\begin{aligned}
& (T(u, \varepsilon))^{(i)}=\left(K^{\prime}(u, \varepsilon)[S(u, \varepsilon)]\right)^{(i)}\left(\hat{\eta}_{i}\right)-\left(S^{\prime}(u, \varepsilon)[K(u, \varepsilon)]\right)^{(i)}\left(\hat{\eta}_{i}\right) \\
= & \left((K(u, \varepsilon))^{(i)}\right)^{\prime}\left(\hat{\eta}_{i}\right)\left[\hat{S}_{i}\right]-\left((S(u, \varepsilon))^{(i)}\right)^{\prime}\left(\hat{\eta}_{i}\right)\left[\hat{K}_{i}\right]
\end{aligned}
$$




$$
=\left(\hat{K}_{N}^{\prime}\left(\hat{\eta}_{N}\right)\left[\hat{S}_{N}\right]\right)_{i}-\left(\hat{S}_{N}^{\prime}\left(\hat{\eta}_{N}\right)\left[\hat{K}_{N}\right]\right)_{i}, 0 \leq i \leq N .
$$

This shows that (2.28) holds. All other results are a direct consequence of (2.28). The proof is completed.

The relation (2.28) implies that the perturbation series (2.1) keeps the Lie product of vector fields invariant. In particular, the second component of (2.28) yields the Lie product property (2.9) of linearized operators. In what follows, we will go on to consider Lax representations and zero curvature representaticns for the perturbation system defined by (2.7). In our formulation below, we will adopt the following notation for the perturbation of a spectral parameter $\lambda$ :

$$
\hat{\lambda}_{N}=\sum_{i=0}^{N} \varepsilon^{i} \mu_{i}, \hat{\mu}_{N}=\left(\mu_{0}, \mu_{1}, \cdots, \mu_{N}\right)^{T},
$$

which is quite similar to the notation for the perturbation of the potential $u$. Here $\mu_{i}, 0 \leq i \leq N$, will be taken as the spectral parameters appearing in the perturbation spectral problems. A customary symbol $\nabla_{x} \lambda, x \in \mathbb{R}^{p}$, will still be used to denote the gradient of the spectral parameter $\lambda$ with respect to $x$.

TheOREM 2.7. Let $K=K(\varepsilon) \in T(M)$ be analytic with respect to $\varepsilon$. Assume that the system $u_{t}=K(u, \varepsilon)$ has an isospectral Lax representation

$$
\left\{\begin{array}{l}
L(u, \varepsilon) \phi=\lambda \phi, \\
\phi_{t}=A(u, \varepsilon) \phi,
\end{array} \quad\left(\nabla_{x} \lambda=0, x \in \mathbb{R}^{p}\right), \text { i.e., }(L(u, \varepsilon))_{t}=[A(u, \varepsilon), L(u, \varepsilon)],\right.
$$

where $L$ and $A$ are two $s \times s$ matrix differential operators being analytic with respect to $u$ and $\varepsilon$. Define the perturbation spectral operator $\hat{L}_{N}$ and the perturbation Lax operator $\hat{A}_{N}$ by

$$
\begin{aligned}
& \left(\operatorname{per}_{N} B\right)\left(\hat{\eta}_{N}\right)=\hat{B}_{N}\left(\hat{\eta}_{N}\right) \\
& =\left[\left(\hat{B}_{N}\left(\hat{\eta}_{N}\right)\right)_{i j}\right]_{i, j=0,1, \cdots, N}=\left[\left.\frac{1}{(i-j) !} \frac{\partial^{i-j} B\left(\hat{u}_{N}, \varepsilon\right)}{\partial \varepsilon^{i-j}}\right|_{\varepsilon=0}\right]_{s(N+1) \times s(N+1)}
\end{aligned}
$$

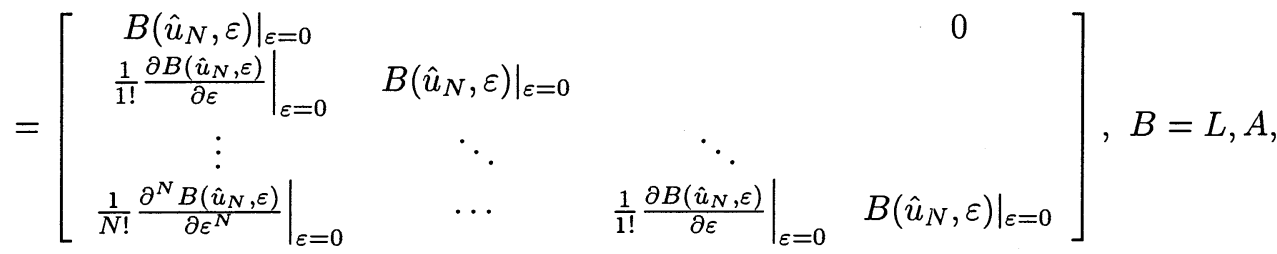

where $\hat{u}_{N}$ is given by (2.1). Then under the condition for the spectral operator $L$ that

$$
\text { if } L^{\prime}\left(\hat{u}_{N}\right)\left[S\left(\hat{u}_{N}\right)\right]=o\left(\varepsilon^{N}\right), S \in T(M) \text {, then } S\left(\hat{u}_{N}\right)=o\left(\varepsilon^{N}\right) \text {, }
$$

the $N$-th order perturbation system $\hat{\eta}_{N t}=\hat{K}_{N}\left(\hat{\eta}_{N}\right)$ defined by (2.7) has the following isospectral Lax representation

$$
\left(\hat{L}_{N}\left(\hat{\eta}_{N}\right)\right)_{t}=\left[\hat{A}_{N}\left(\hat{\eta}_{N}\right), \hat{L}_{N}\left(\hat{\eta}_{N}\right)\right],
$$

which is the compatibility condition of the following perturbation spectral problem

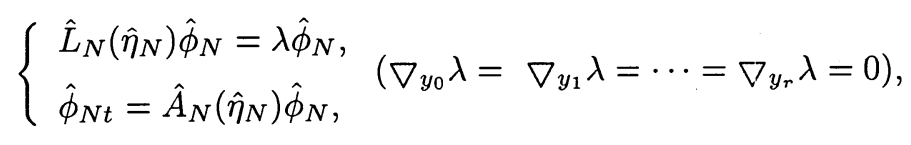


or the following perturbation spectral problem

$$
\left\{\begin{array}{l}
\hat{L}_{N}\left(\hat{\eta}_{N}\right) \hat{\phi}_{N}=\Lambda \hat{\phi}_{N} \\
\hat{\phi}_{N t}=\hat{A}_{N}\left(\hat{\eta}_{N}\right) \hat{\phi}_{N}
\end{array}\right.
$$

where the matrix $\Lambda$ reads as

$$
\Lambda=\left[\begin{array}{cccc}
\mu_{0} I_{s} & & & \\
\mu_{1} I_{s} & \mu_{0} I_{s} & & \\
\vdots & \ddots & \ddots & \\
\mu_{N} I_{s} & \cdots & \mu_{1} I_{s} & \mu_{0} I_{s}
\end{array}\right], I_{s}=\operatorname{diag} \underbrace{1,1, \cdots, 1}_{s})
$$

with the spectral parameter $\mu_{i}, 0 \leq i \leq N$, satisfying

$$
\sum_{k+l=i} \nabla y_{k} \mu_{l}=0,0 \leq i \leq N
$$

Proof. We first observe that the perturbed system

$$
\hat{u}_{N t}=K\left(\hat{u}_{N}, \varepsilon\right)+o\left(\varepsilon^{N}\right)
$$

which engenders precisely the perturbation system $\hat{\eta}_{N t}=\hat{K}_{N}\left(\hat{\eta}_{N}\right)$ defined by (2.7). Noting that $L(u, \varepsilon), A(u, \varepsilon)$ are analytic with respect to $u$ and $\varepsilon$, it follows from (2.31) that (2.38) is equivalent to the following

$$
\left.\frac{\partial^{k}}{\partial \varepsilon^{k}}\right|_{\varepsilon=0}\left(\left(L\left(\hat{u}_{N}, \varepsilon\right)\right)_{t}-\left[A\left(\hat{u}_{N}, \varepsilon\right), L\left(\hat{u}_{N}, \varepsilon\right)\right]\right)=0,0 \leq k \leq N
$$

by use of (2.32).

What we want to prove next is that (2.39) is equivalent to (2.33). Let us compute the elements of the differential operator matrix $\left[\hat{A}_{N}\left(\hat{\eta}_{N}\right), \hat{L}_{N}\left(\hat{\eta}_{N}\right)\right]$. It is obvious that $\left[\hat{A}_{N}\left(\hat{\eta}_{N}\right), \hat{L}_{N}\left(\hat{\eta}_{N}\right)\right]$ is lower triangular, that is to say,

$$
\left(\left[\hat{A}_{N}\left(\hat{\eta}_{N}\right), \hat{L}_{N}\left(\hat{\eta}_{N}\right)\right]\right)_{i j}=0,0 \leq i<j \leq N .
$$

For the other part of $\left[\hat{A}_{N}\left(\hat{\eta}_{N}\right), \hat{L}_{N}\left(\hat{\eta}_{N}\right)\right]$, we can compute that

$$
\begin{aligned}
\left(\hat{A}_{N}\left(\hat{\eta}_{N}\right) \hat{L}_{N}\left(\hat{\eta}_{N}\right)\right)_{i j} & =\left.\left.\sum_{k=j}^{i} \frac{1}{(i-k) !} \frac{\partial^{i-k} A\left(\hat{u}_{N}, \varepsilon\right)}{\partial \varepsilon^{i-k}}\right|_{\varepsilon=0} \frac{1}{(k-j) !} \frac{\partial^{k-j} L\left(\hat{u}_{N}, \varepsilon\right)}{\partial \varepsilon^{k-j}}\right|_{\varepsilon=0} \\
& =\left.\left.\frac{1}{(i-j) !} \sum_{k=j}^{i}\left(\begin{array}{c}
i-j \\
i-k
\end{array}\right) \frac{\partial^{i-k} A\left(\hat{u}_{N}, \varepsilon\right)}{\partial \varepsilon^{i-k}}\right|_{\varepsilon=0} \frac{\partial^{k-j} L\left(\hat{u}_{N}, \varepsilon\right)}{\partial \varepsilon^{k-j}}\right|_{\varepsilon=0} \\
& =\left.\frac{1}{(i-j) !} \frac{\partial^{i-j} A\left(\hat{u}_{N}, \varepsilon\right) L\left(\hat{u}_{N}, \varepsilon\right)}{\partial \varepsilon^{i-j}}\right|_{\varepsilon=0}, 0 \leq j \leq i \leq N,
\end{aligned}
$$

where the $\left(\begin{array}{l}i \\ j\end{array}\right)$ are the binomial coefficients. In the same way, we can obtain

$$
\left(\hat{L}_{N}\left(\hat{\eta}_{N}\right) \hat{A}_{N}\left(\hat{\eta}_{N}\right)\right)_{i j}=\left.\frac{1}{(i-j) !} \frac{\partial^{i-j} L\left(\hat{u}_{N}, \varepsilon\right) A\left(\hat{u}_{N}, \varepsilon\right)}{\partial \varepsilon^{i-j}}\right|_{\varepsilon=0}, 0 \leq j \leq i \leq N .
$$


Therefore we arrive at

$$
\left(\left[\hat{A}_{N}\left(\hat{\eta}_{N}\right), \hat{L}_{N}\left(\hat{\eta}_{N}\right)\right]\right)_{i j}=\left.\frac{1}{(i-j) !} \frac{\partial^{i-j}\left[A\left(\hat{u}_{N}, \varepsilon\right), L\left(\hat{u}_{N}, \varepsilon\right)\right]}{\partial \varepsilon^{i-j}}\right|_{\varepsilon=0}, 0 \leq j \leq i \leq N .
$$

Now it is easy to find that (2.39) is equivalent to (2.33). Therefore the perturbation system defined by (2.7) has the Lax representation (2.33).

Let us now turn to the perturbation spectral problems (2.34) and (2.35). Obviously, the compatibility condition of the perturbation spectral problem (2.34) is the Lax equation (2.33), since the spectral parameter $\lambda$ doesn't vary whatever the spatial variables change. Therefore let us consider the compatibility condition of the perturbation spectral problem (2.35). First, we want to prove that

$$
\Lambda \hat{A}_{N}\left(\hat{\eta}_{N}\right)=\hat{A}_{N}\left(\hat{\eta}_{N}\right) \Lambda
$$

if the spectral parameters $\mu_{i}, 0 \leq i \leq N$, satisfy (2.37). Notice that the condition (2.37) on the spectral parameters $\mu_{i}, 0 \leq i \leq N$, is required by

$$
\hat{\nabla}_{x} \hat{\lambda}_{N}=\mathrm{o}\left(\varepsilon^{N}\right), \hat{\nabla}_{x}=\sum_{i=0}^{r} \varepsilon^{i} \nabla_{y_{i}}, \hat{\lambda}_{N}=\sum_{i=0}^{N} \varepsilon^{i} \mu_{i}
$$

which is a perturbation version of $\nabla_{x} \lambda=0$. Therefore we have

$$
A\left(\hat{u}_{N}, \varepsilon, \hat{\nabla}_{x}\right) \hat{\lambda}_{N}=\hat{\lambda}_{N} A\left(\hat{u}_{N}, \varepsilon, \hat{\nabla}_{x}\right)+\mathrm{o}\left(\varepsilon^{N}\right) .
$$

This guarantees that

$$
\left.\frac{1}{(i-j) !} \frac{\partial^{i-j}}{\partial \varepsilon^{i-j}}\right|_{\varepsilon=0}\left(A\left(\hat{u}_{N}, \varepsilon, \hat{\nabla}_{x}\right) \hat{\lambda}_{N}\right)=\left.\frac{1}{(i-j) !} \frac{\partial^{i-j}}{\partial \varepsilon^{i-j}}\right|_{\varepsilon=0}\left(\hat{\lambda}_{N} A\left(\hat{u}_{N}, \varepsilon, \hat{\nabla}_{x}\right)\right)
$$

for $0 \leq j \leq i \leq N$, which exactly means that the equality (2.40) holds. Now we can compute from (2.35) that

$$
\begin{gathered}
\left(\hat{L}_{N}\left(\hat{\eta}_{N}\right)\right)_{t} \hat{\phi}_{N}+\hat{L}_{N}\left(\hat{\eta}_{N}\right) \hat{A}_{N}\left(\hat{\eta}_{N}\right) \hat{\phi}_{N}=\Lambda \hat{A}_{N}\left(\hat{\eta}_{N}\right) \hat{\phi}_{N} \\
=\hat{A}_{N}\left(\hat{\eta}_{N}\right) \Lambda \hat{\phi}_{N}=\hat{A}_{N}\left(\hat{\eta}_{N}\right) \hat{L}_{N}\left(\hat{\eta}_{N}\right) \hat{\phi}_{N} .
\end{gathered}
$$

It follows that the compatibility condition of the perturbation spectral problem (2.35) is also the Lax equation (2.33). The proof is completed.

The perturbation spectral operator $\hat{L}_{N}$ is very similar to the perturbation recursion operator $\hat{\Phi}_{N}$, in spite of different orders of matrices. Actually, we may take any recursion operator $\Phi$ as a spectral operator and the system $u_{t}=K(u)$ can have a Lax representation $\Phi_{t}=\left[\Phi, K^{\prime}\right]$. This Lax representation is usually non-local, because most recursion operators are intego-differential. We also remark that two perturbation spectral problems above are represented for the same perturbation system defined by (2.7), which involve different conditions on the spectral parameters. For the case of

$$
\hat{u}_{N}=\sum_{i=0}^{N} \varepsilon^{i} \eta_{i}(x, y, t)=\sum_{i=0}^{N} \varepsilon^{i} \eta_{i}(x, \varepsilon x, t), x \in \mathbb{R},
$$

the condition (2.37) can be reduced to

$$
\mu_{0 x}=0, \mu_{i x}+\mu_{i-1, y}=0,1 \leq i \leq N
$$


In the following theorem, a similar result is shown for zero curvature representations of the perturbation systems defined by (2.7).

Theorem 2.8. Let $K=K(\varepsilon) \in T(M)$ be analytic with respect to $\varepsilon$. Assume that the initial system $u_{t}=K(u, \varepsilon)$ has an isospectral zero curvature representation

$$
\begin{gathered}
\left\{\begin{array}{c}
\phi_{x}=U(u, \lambda, \varepsilon) \phi, \\
\phi_{t}=V(u, \lambda, \varepsilon) \phi,
\end{array} \quad\left(\lambda_{x}=0, x \in \mathbb{R}\right),\right. \\
\text { i.e., }(U(u, \lambda, \varepsilon))_{t}-(V(u, \lambda, \varepsilon))_{x}+[U(u, \lambda, \varepsilon), V(u, \lambda, \varepsilon)]=0,
\end{gathered}
$$

where $U$ and $V$ are two $s \times s$ matrix differential (sometimes multiplication) operators being analytic with respect to $u, \lambda$ and $\varepsilon$. Define two perturbation matrix differential operators $\hat{U}_{N}$ and $\hat{V}_{N}$ by

$$
\begin{aligned}
& \left(\operatorname{per}_{N} W\right)\left(\hat{\eta}_{N}\right)=\hat{W}_{N}\left(\hat{\eta}_{N}, \hat{\mu}_{N}\right)=\hat{W}_{N}\left(\hat{\eta}_{N}\right) \\
& =\left[\left(\hat{W}_{N}\left(\hat{\eta}_{N}\right)\right)_{i j}\right]_{i, j=0,1, \cdots, N}=\left[\left.\frac{1}{(i-j) !} \frac{\partial^{i-j} W\left(\hat{u}_{N}, \hat{\lambda}_{N}\right), \varepsilon}{\partial \varepsilon^{i-j}}\right|_{\varepsilon=0}\right]_{s(N+1) \times s(N+1)} \\
& =\left[\begin{array}{ccc}
\left.W\left(\hat{\eta}_{N}, \hat{\lambda}_{N}, \varepsilon\right)\right|_{\varepsilon=0} & & 0 \\
\left.\frac{1}{1 !} \frac{\partial W\left(\hat{u}_{N}, \hat{\lambda}_{N}, \varepsilon\right)}{\partial \varepsilon}\right|_{\varepsilon=0} & \left.W\left(\hat{\eta}_{N}, \hat{\lambda}_{N}, \varepsilon\right)\right|_{\varepsilon=0} & \\
\vdots & \ddots & \ddots \\
\left.\frac{1}{N !} \frac{\partial^{N} W\left(\hat{u}_{N}, \hat{\lambda}_{N}, \varepsilon\right)}{\partial \varepsilon^{N}}\right|_{\varepsilon=0} & \cdots & \left.\left.\frac{1}{1 !} \frac{\partial W\left(\hat{u}_{N}, \hat{\lambda}_{N}, \varepsilon\right)}{\partial \varepsilon}\right|_{\varepsilon=0} W\left(\hat{\eta}_{N}, \hat{\lambda}_{N}, \varepsilon\right)\right|_{\varepsilon=0}
\end{array}\right],
\end{aligned}
$$

where $W=U$ or $V$, and $\hat{u}_{N}$ and $\hat{\lambda}_{N}$ are given by (2.41) and (2.30). Then under the condition for the spectral operator $U$ that

$$
\text { if } U^{\prime}\left(\hat{u}_{N}\right)\left[S\left(\hat{u}_{N}\right)\right]=o\left(\varepsilon^{N}\right), S \in T(M) \text {, then } S\left(\hat{u}_{N}\right)=o\left(\varepsilon^{N}\right),
$$

the $N$-th order perturbation system $\hat{\eta}_{N t}=\hat{K}_{N}\left(\hat{\eta}_{N}\right)$ defined by (2.7) has the following isospectral zero curvature representation

$$
\left\{\begin{array}{l}
\sum_{i=0}^{r} \Pi^{i} \hat{\phi}_{N y_{i}}=\hat{U}_{N}\left(\hat{\eta}_{N}, \hat{\mu}_{N}\right) \hat{\phi}_{N} \\
\hat{\phi}_{N t}=\hat{V}_{N}\left(\hat{\eta}_{N}, \hat{\mu}_{N}\right) \hat{\phi}_{N}
\end{array}\right.
$$

$$
\text { i.e., }\left(\hat{U}_{N}\left(\hat{\eta}_{N}\right)\right)_{t}-\sum_{i=0}^{r} \Pi^{i}\left(\hat{V}_{N}\left(\hat{\eta}_{N}\right)\right)_{y_{i}}+\left[\hat{U}_{N}\left(\hat{\eta}_{N}\right), \hat{V}_{N}\left(\hat{\eta}_{N}\right)\right]=0
$$

where the matrix $\Pi$ is defined by

$$
\Pi=\left[\begin{array}{cc}
0 & 0 \\
I_{s N} & 0
\end{array}\right]_{s(N+1) \times s(N+1)}, I_{s N}=\operatorname{diag}(\underbrace{I_{s}, \cdots, I_{s}}_{N})=\operatorname{diag}(\underbrace{1, \cdots, 1}_{s N})
$$


and the spectral parameters $\mu_{i}, 0 \leq i \leq N$, satisfy

$$
\sum_{k+l=i} \partial_{y_{k}} \mu_{l}=0,0 \leq i \leq N
$$

Proof. Note that by use of (2.45), the zero curvature equation

$$
\left((U(u, \lambda, \varepsilon))_{t}-(V(u, \lambda, \varepsilon))_{x}+[U(u, \lambda, \varepsilon), V(u, \lambda, \varepsilon)]=0\right.
$$

for the system $u_{t}=K(u, \varepsilon)$ yields an equivalent representation

$$
\begin{aligned}
& \left(U\left(\hat{u}_{N}, \hat{\lambda}_{N}, \varepsilon\right)\right)_{t}-\sum_{i=0}^{r} \varepsilon^{i}\left(V\left(\hat{u}_{N}, \hat{\lambda}_{N}, \varepsilon\right)\right)_{y_{i}}+\left[U\left(\hat{u}_{N}, \hat{\lambda}_{N}, \varepsilon\right), V\left(\hat{u}_{N}, \hat{\lambda}_{N}, \varepsilon\right)\right] \\
\equiv & U^{\prime}\left(\hat{u}_{N}\right)\left[K\left(\hat{u}_{N}, \varepsilon\right)\right]-\sum_{i=0}^{r} \varepsilon^{i}\left(V\left(\hat{u}_{N}, \hat{\lambda}_{N}, \varepsilon\right)\right)_{y_{i}}+\left[U\left(\hat{u}_{N}, \hat{\lambda}_{N}, \varepsilon\right), V\left(\hat{u}_{N}, \hat{\lambda}_{N}, \varepsilon\right)\right] \\
\equiv & o\left(\varepsilon^{N}\right) \quad\left(\bmod \varepsilon^{N}\right)
\end{aligned}
$$

for the perturbation system $\hat{\eta}_{N t}=\hat{K}_{N}\left(\hat{\eta}_{N}\right)$. In order to recover $\hat{u}_{N t}=K\left(\hat{u}_{N}, \varepsilon\right)+$ $\mathrm{o}\left(\varepsilon^{N}\right)$ from (2.50), we need to keep the spectral property $\lambda_{x}=\partial_{x} \lambda=0$ under the perturbation up to a precision $o\left(\varepsilon^{N}\right)$. This requires

$$
\hat{\partial}_{x} \hat{\lambda}_{N}=\mathrm{o}\left(\varepsilon^{N}\right), \hat{\partial}_{x}=\sum_{i=0}^{r} \varepsilon^{i} \partial_{y_{i}}, \hat{\lambda}_{N}=\sum_{i=0}^{N} \varepsilon^{i} \mu_{i},
$$

which generates (2.49). Similar to the proof of Theorem 2.7, differentiating the above equation (2.50) with respect to $\varepsilon$ up to $N$ times leads to the zero curvature equation (2.47), and conversely, we have (2.50) if (2.47) holds. Therefore the perturbation system $\hat{\eta}_{N t}=\hat{K}_{N}\left(\hat{\eta}_{N}\right)$ has an isospectral zero curvature representation (2.47).

The other thing that we need to prove is that the zero curvature equation (2.47) is exactly the compatibility condition of the perturbation spectral problem (2.46). From the first system of (2.46), we have

$$
\sum_{i=0}^{r} \Pi^{i} \hat{\phi}_{N y_{i} t}=\hat{U}_{N t} \hat{\phi}_{N}+\hat{U}_{N} \hat{\phi}_{N t} .
$$

From the second system of (2.46), we obtain

$$
\hat{\phi}_{N t y_{i}}=\hat{V}_{N y_{i}} \hat{\phi}_{N}+\hat{V}_{N} \hat{\phi}_{N y_{i}}, 0 \leq i \leq r .
$$

A combination of the above equalities yields

$$
\sum_{i=0}^{r} \Pi^{i}\left(\hat{V}_{N y_{i}} \hat{\phi}_{N}+\hat{V}_{N} \hat{\phi}_{N y_{i}}\right)=\hat{U}_{N t} \hat{\phi}_{N}+\hat{U}_{N} \hat{V}_{N} \hat{\phi}_{N}
$$

On the other hand, we have

$$
\sum_{i=0}^{N} \Pi^{i} \hat{V}_{N} \hat{\phi}_{N y_{i}}=\sum_{i=0}^{N} \hat{V}_{N} \Pi^{i} \hat{\phi}_{N y_{i}}=\hat{V}_{N} \hat{U}_{N} \hat{\phi}_{N}
$$


by using $\Pi \hat{V}_{N}=\hat{V}_{N} \Pi$ and the first system of (2.46). It follows from (2.51) and (2.52) that the zero curvature equation (2.47) is the compatibility condition of the perturbation spectral problem (2.46). The proof is completed.

If we consider the specific case of the perturbation defined by $(2.41)$, then the perturbation spectral problem and the perturbation zero curvature equation, defined by (2.46) and (2.47), will be simplified to

$$
\left\{\begin{array}{l}
\hat{\phi}_{N x}+\Pi \hat{\phi}_{N y}=\hat{U}_{N}\left(\hat{\eta}_{N}, \hat{\mu}_{N}\right) \hat{\phi}_{N} \\
\hat{\phi}_{N t}=\hat{V}_{N}\left(\hat{\eta}_{N}, \hat{\mu}_{N}\right) \hat{\phi}_{N}
\end{array}\right.
$$

and

$$
\left(\hat{U}_{N}\left(\hat{\eta}_{N}\right)\right)_{t}-\left(\hat{V}_{N}\left(\hat{\eta}_{N}\right)\right)_{x}-\Pi\left(\hat{V}_{N}\left(\hat{\eta}_{N}\right)\right)_{y}+\left[\hat{U}_{N}\left(\hat{\eta}_{N}\right), \hat{V}_{N}\left(\hat{\eta}_{N}\right)\right]=0
$$

respectively. The involved spectral parameters $\mu_{i}, 0 \leq i \leq N$, need to satisfy a reduction (2.42) of the general condition (2.49).

TheOREM 2.9. Let $K=K(\varepsilon) \in T(M)$ be analytic with respect to $\varepsilon$. Assume that the initial system $u_{t}=K(u, \varepsilon)$ possesses a Hamiltonian formulation

$$
u_{t}=K(u, \varepsilon)=J(u, \varepsilon) \frac{\delta \tilde{H}}{\delta u}(u, \varepsilon)
$$

where $J: T^{*}(M) \rightarrow T(M)$ is a Hamiltonian operator and $\tilde{H} \in C^{\infty}(M)$ is a Hamiltonian functional. Then the perturbation system $\hat{\eta}_{N t}=\hat{K}_{N}\left(\hat{\eta}_{N}\right)$ defined by (2.7) also possesses a Hamiltonian formulation

$$
\hat{\eta}_{N t}=\hat{K}_{N}\left(\hat{\eta}_{N}\right)=\hat{J}_{N}\left(\hat{\eta}_{N}\right) \frac{\delta\left(p e r_{N} \tilde{H}\right)}{\delta \hat{\eta}_{N}}\left(\hat{\eta}_{N}\right)
$$

where the Hamiltonian operator $\hat{J}_{N}\left(\hat{\eta}_{N}\right)$ is determined by (2.17) and the Hamiltonian functional $\operatorname{per}_{N} \tilde{H}=\hat{\tilde{H}}_{N} \in C^{\infty}\left(\hat{M}_{N}\right)$ is defined by

$$
\left(\operatorname{per}_{N} \tilde{H}\right)\left(\hat{\eta}_{N}\right)=\hat{\tilde{H}}_{N}\left(\hat{\eta}_{N}\right)=\left.\frac{1}{N !} \frac{\partial^{N} \tilde{H}\left(\hat{u}_{N}, \varepsilon\right)}{\partial \varepsilon^{N}}\right|_{\varepsilon=0}
$$

The corresponding Poisson bracket has the property

$$
\left\{\operatorname{per}_{N} \tilde{H}_{1}, \operatorname{per}_{N} \tilde{H}_{2}\right\}_{\hat{J}_{N}}=\operatorname{per}_{N}\left\{\tilde{H}_{1}, \tilde{H}_{2}\right\}_{J}, \tilde{H}_{1}, \tilde{H}_{2} \in C^{\infty}(M) .
$$

Moreover the perturbation systems $\hat{\eta}_{N t}=\hat{K}_{N}\left(\hat{\eta}_{N}\right)$ defined by (2.7) possesses a multiHamiltonian formulation

$$
\hat{\eta}_{N t}=\hat{K}_{N}\left(\hat{\eta}_{N}\right)=\hat{J}_{1 N}\left(\hat{\eta}_{N}\right) \frac{\delta\left(\operatorname{per}_{N} \tilde{H}_{1}\right)}{\delta \hat{\eta}_{N}}\left(\hat{\eta}_{N}\right)=\cdots=\hat{J}_{m N}\left(\hat{\eta}_{N}\right) \frac{\delta\left(\operatorname{per}_{N} \tilde{H}_{m}\right)}{\delta \hat{\eta}_{N}}\left(\hat{\eta}_{N}\right),
$$

if $u_{t}=K(u, \varepsilon)$ possesses an analogous multi-Hamiltonian formulation

$$
u_{t}=K(u, \varepsilon)=J_{1}(u, \varepsilon) \frac{\delta \tilde{H}_{1}}{\delta u}(u, \varepsilon)=\cdots=J_{m}(u, \varepsilon) \frac{\delta \tilde{H}_{m}}{\delta u}(u, \varepsilon) .
$$


Proof. Assume that $\gamma(\varepsilon)=\frac{\delta \tilde{H}}{\delta u}(\varepsilon) \in T^{*}(M)$. Let us observe that

$$
\begin{aligned}
\eta_{i t} & =\left.\frac{1}{i !} \frac{\partial^{i}\left(J\left(\hat{u}_{N}, \varepsilon\right) \gamma\left(\hat{u}_{N}, \varepsilon\right)\right)}{\partial \varepsilon^{i}}\right|_{\varepsilon=0} \\
& =\left.\left.\sum_{j=0}^{i} \frac{1}{j !(i-j) !} \frac{\partial^{i-j} J\left(\hat{u}_{N}, \varepsilon\right)}{\partial \varepsilon^{i-j}}\right|_{\varepsilon=0} \frac{\partial^{j} \gamma\left(\hat{u}_{N}, \varepsilon\right)}{\partial \varepsilon^{j}}\right|_{\varepsilon=0}, 0 \leq i \leq N .
\end{aligned}
$$

Thus, noting the structure of $\hat{J}_{N}$, we can represent the perturbation system as follows

$$
\hat{\eta}_{N t}=\hat{K}_{N}\left(\hat{\eta}_{N}\right)=\hat{J}_{N}\left(\hat{\eta}_{N}\right) \hat{\gamma}_{N}\left(\hat{\eta}_{N}\right)
$$

where the cotangent vector field $\hat{\gamma}_{N} \in T^{*}\left(\hat{M}_{N}\right)$ reads as

$$
\begin{aligned}
\hat{\gamma}_{N}\left(\hat{\eta}_{N}\right)= & \left.\frac{1}{N !} \frac{\partial^{N} \gamma^{T}\left(\hat{u}_{N}, \varepsilon\right)}{\partial \varepsilon^{N}}\right|_{\varepsilon=0},\left.\frac{1}{(N-1) !} \frac{\partial^{N-1} \gamma^{T}\left(\hat{u}_{N}, \varepsilon\right)}{\partial \varepsilon^{N-1}}\right|_{\varepsilon=0}, \\
& \left.\cdots,\left.\frac{1}{1 !} \frac{\partial \gamma^{T}\left(\hat{u}_{N}, \varepsilon\right)}{\partial \varepsilon}\right|_{\varepsilon=0},\left.\gamma^{T}\left(\hat{u}_{N}, \varepsilon\right)\right|_{\varepsilon=0}\right)^{T} .
\end{aligned}
$$

Let us check whether this cotangent vector field $\hat{\gamma}_{N}$ is a gradient field. If it is gradient, the corresponding potential functional has to be the following

$$
\begin{aligned}
& \int_{0}^{1}<\hat{\gamma}_{N}\left(\lambda \hat{\eta}_{N}\right), \hat{\eta}_{N}>d \lambda \\
= & \int_{0}^{1} \sum_{i=0}^{N} \frac{1}{i !}<\left.\frac{\partial^{i} \gamma\left(\lambda \hat{u}_{N}, \varepsilon\right)}{\partial \varepsilon^{i}}\right|_{\varepsilon=0}, \eta_{N-i}>d \lambda \\
= & \left.\frac{1}{N !} \frac{\partial^{N}}{\partial \varepsilon^{N}}\right|_{\varepsilon=0} \int_{0}^{1}<\gamma\left(\lambda \hat{u}_{N}, \varepsilon\right), \hat{u}_{N}>d \lambda=\left.\frac{1}{N !} \frac{\partial^{N} \tilde{H}\left(\hat{u}_{N}, \varepsilon\right)}{\partial \varepsilon^{N}}\right|_{\varepsilon=0} .
\end{aligned}
$$

The cotangent vector field $\hat{\gamma}_{N}$ is indeed a gradient field, because we can show that

$$
\hat{\gamma}_{N}\left(\hat{\eta}_{N}\right)=\frac{\delta\left(\operatorname{per}_{N} \tilde{H}\right)}{\delta \hat{\eta}_{N}}\left(\hat{\eta}_{N}\right) .
$$

According to Definition 1.1 and using Lemma 2.1, for any $S_{i} \in T\left(M\left(\eta_{i}\right)\right)$ we can compute that

$$
\begin{aligned}
& <\frac{\delta}{\delta \eta_{i}}\left(\left.\frac{1}{N !} \frac{\partial^{N} \tilde{H}\left(\hat{u}_{N}, \varepsilon\right)}{\partial \varepsilon^{N}}\right|_{\varepsilon=0}\right), S_{i}\left(\eta_{i}\right)>=\left(\left.\frac{1}{N !} \frac{\partial^{N} \tilde{H}\left(\hat{u}_{N}, \varepsilon\right)}{\partial \varepsilon^{N}}\right|_{\varepsilon=0}\right)^{\prime}\left(\eta_{i}\right)\left[S_{i}\left(\eta_{i}\right)\right] \\
= & \left.\frac{1}{N !} \frac{\partial^{N}}{\partial \varepsilon^{N}}\right|_{\varepsilon=0} \tilde{H}^{\prime}\left(\hat{u}_{N}, \varepsilon\right)\left[\varepsilon^{i} S_{i}\left(\eta_{i}\right)\right]=\left.\frac{1}{(N-i) !} \frac{\partial^{N-i}}{\partial \varepsilon^{N-i}}\right|_{\varepsilon=0} \tilde{H}^{\prime}\left(\hat{u}_{N}, \varepsilon\right)\left[S_{i}\left(\eta_{i}\right)\right] \\
= & \left.\frac{1}{(N-i) !} \frac{\partial^{N-i}}{\partial \varepsilon^{N-i}}\right|_{\varepsilon=0}<\frac{\delta \tilde{H}}{\delta \hat{u}_{N}}\left(\hat{u}_{N}, \varepsilon\right), S_{i}\left(\eta_{i}\right)> \\
= & \left.\frac{1}{(N-i) !} \frac{\partial^{N-i}}{\partial \varepsilon^{N-i}}\right|_{\varepsilon=0}<\gamma\left(\hat{u}_{N}, \varepsilon\right), S_{i}\left(\eta_{i}\right)> \\
= & <\left.\frac{1}{(N-i) !} \frac{\partial^{N-i} \gamma\left(\hat{u}_{N}, \varepsilon\right)}{\partial \varepsilon^{N-i}}\right|_{\varepsilon=0}, S_{i}\left(\eta_{i}\right)>, 0 \leq i \leq N .
\end{aligned}
$$


This equality implies that (2.60) holds. It follows that the perturbation system (2.58) is a Hamiltonian system.

Let us now turn to the property (2.57) for the Poisson bracket. Set $\gamma_{1}(\varepsilon)=$ $\frac{\delta \tilde{H}_{1}}{\delta u}(\varepsilon), \gamma_{2}(\varepsilon)=\frac{\delta \tilde{H}_{2}}{\delta u}(\varepsilon) \in T^{*}(M)$. In virtue of $(2.60)$, we can make the computation

$$
\begin{aligned}
& \left\{\operatorname{per}_{N} \tilde{H}_{1}, \operatorname{per}_{N} \tilde{H}_{2}\right\}_{\hat{J}_{N}}\left(\hat{\eta}_{N}\right)=<\frac{\delta\left(\operatorname{per}_{N} \tilde{H}_{1}\right)}{\delta \hat{\eta}_{N}}\left(\hat{\eta}_{N}\right), \hat{J}_{N}\left(\hat{\eta}_{N}\right) \frac{\delta\left(\operatorname{per}_{N} \tilde{H}_{2}\right)}{\delta \hat{\eta}_{N}}\left(\hat{\eta}_{N}\right)> \\
= & \sum_{i=0}^{N}<\left.\frac{1}{(N-i) !} \frac{\partial^{N-i} \gamma_{1}\left(\hat{u}_{N}, \varepsilon\right)}{\partial \varepsilon^{N-i}}\right|_{\varepsilon=0}, \sum_{j=N-i}^{N} \frac{1}{(i+j-N) !} \times \\
= & \sum_{i=0}^{N}<\left.\left.\frac{1}{(N-i) !} \frac{\partial^{i+j-N} J\left(\hat{u}_{N}, \varepsilon\right)}{\partial \varepsilon^{i+j-N}}\right|_{\varepsilon=0} \frac{1}{(N-j) !} \frac{\partial^{N-j} \gamma_{2}\left(\hat{u}_{N}, \varepsilon\right)}{\partial \varepsilon^{N-j}}\right|_{\varepsilon=0}> \\
= & \left.\frac{1}{N !} \frac{\partial^{N}}{\partial \varepsilon^{N}}\right|_{\varepsilon=0}<\gamma_{1}\left(\hat{u}_{N}, \varepsilon\right), J\left(\hat{u}_{N}, \varepsilon\right) \gamma_{2}\left(\hat{u}_{N}, \varepsilon\right)>=\left(\operatorname{per}_{N}\left\{\tilde{H}_{1}, \tilde{H}_{2}\right\}_{J}\right)\left(\hat{\eta}_{N}\right) .
\end{aligned}
$$

This shows that the property (2.57) holds for the Poisson bracket.

Further, noting the structure of the perturbation Hamiltonian operators, a multiHamiltonian formulation may readily be established for the perturbation system. Therefore the proof is completed.

We should realize that two formulas (2.56) and (2.59) provide the explicit structures for the perturbation Hamiltonian functionals and the perturbation cotangent vector fields. The whole theory above can be applied to all soliton hierarchies and thus various interesting perturbation systems including higher dimensional integrable couplings may be presented. In the next section, we will however be only concerned with an application of the theory to the KdV soliton hierarchy.

3. Application to the KdV hierarchy. Let us consider the case of the KdV hierarchy

$$
u_{t}=K_{n}=K_{n}(u)=(\Phi(u))^{n} u_{x}, \Phi=\Phi(u)=\partial_{x}^{2}+2 u_{x} \partial_{x}^{-1}+4 u, n \geq 0 .
$$

Except the first linear equation $u_{t}=u_{x}$, each equation $u_{t}=K(u)(n \geq 1)$ can be written as the following bi-Hamiltonian equation [15]

$$
u_{t}=K_{n}=J \frac{\delta \tilde{H}_{n}}{\delta u}=M \frac{\delta \tilde{H}_{n-1}}{\delta u}
$$

The corresponding Hamiltonian pair and Hamiltonian functionals read as

$$
\begin{aligned}
& J=\partial_{x}, M=M(u)=\partial_{x}^{3}+2\left(\partial_{x} u+u \partial_{x}\right), \\
& \tilde{H}_{n}=\int H_{n} d x, H_{n}=H_{n}(u)=\int_{0}^{1} u f_{n}(\lambda u) d \lambda, f_{n}=\Psi^{n} u, n \geq 0,
\end{aligned}
$$

where $\Psi=\Phi^{\dagger}=\partial_{x}^{2}+4 u-2 \partial_{x}^{-1} u_{x}$. Therefore each equation in the KdV hierarchy (3.1) has infinitely many commuting symmetries $\left\{K_{m}\right\}_{m=0}^{\infty}$ and conserved densities $\left\{H_{m}\right\}_{m=0}^{\infty}$.

The second equation in the hierarchy (3.1) gives the following $\mathrm{KdV}$ equation

$$
u_{t}=u_{x x x}+6 u u_{x}
$$


which serves as a well-known model of soliton phenomena. Its many remarkable properties were reviewed by Miura [17]. In our discussion, we are concerned only with bi-Hamiltonian formulations and consequent symmetries and conserved densities. The bi-Hamiltonian formulation of the $\mathrm{KdV}$ equation (3.5) can be written down

$$
u_{t}=J \frac{\delta \tilde{H}_{1}}{\delta u}=M \frac{\delta \tilde{H}_{0}}{\delta u}
$$

with two Hamiltonian functionals

$$
\tilde{H}_{0}=\int H_{0} d x=\int \frac{1}{2} u^{2} d x, \tilde{H}_{1}=\int H_{1} d x=\int\left(\frac{1}{2} u u_{x x}+u^{3}\right) d x .
$$

It has also an isospectral zero curvature representation $U_{t}-V_{x}+[U, V]=0$ with

$$
U=\left[\begin{array}{cc}
0 & -u-\lambda \\
1 & 0
\end{array}\right], V=\left[\begin{array}{cc}
u_{x} & -u_{x x}-2 u^{2}+2 \lambda u+4 \lambda^{2} \\
2 u-4 \lambda & -u_{x}
\end{array}\right]
$$

where $\lambda$ is a spectral parameter (see [18] for more information). These two properties will be used to construct bi-Hamiltonian formulations and zero curvature representations for the related perturbation systems.

In order to apply the general idea of constructing integrable couplings to the $\mathrm{KdV}$ equations, let us start from the following perturbed equation

$$
u_{t}=K^{\mathrm{per}}(u)=\sum_{i=0}^{\infty} \alpha_{i} \varepsilon^{i} S_{i}(u)
$$

where $\alpha_{i}$ are arbitrary constants and the $S_{i}$ are taken from zero function and $K_{n}, n \geq$ 0 , so that the series (3.9) terminates. To obtain integrable couplings of the $n$-th order $\mathrm{KdV}$ equation $u_{t}=K_{n}$, we need to fix $S_{0}=K_{n}$. Various integrable couplings can be generated by making the perturbation defined by (2.1). In what follows, we would only like to present some illustrative examples.

3.1. Standard perturbation systems. First of all, let us choose the $n$-th order $\mathrm{KdV}$ equation itself as an initial equation:

$$
u_{t}=K^{\mathrm{per}}(u)=K_{n}(u)
$$

for each $n \geq 1$. In this case, the single scale perturbation $\hat{u}_{N}=\sum_{i=0}^{N} \varepsilon^{i} \eta_{i}(x, t)$ leads to a type of integrable couplings:

$$
\hat{\eta}_{N t}=\hat{K}_{n N}\left(\hat{\eta}_{N}\right), N \geq 0,
$$

which are called the standard perturbation systems of $u_{t}=K_{n}$ and have been discussed in $[19,20]$. These systems have the following bi-Hamiltonian formulations $[20]$

$$
\hat{\eta}_{N t}=\hat{K}_{n N}\left(\hat{\eta}_{N}\right)=\hat{\Phi}_{N}^{n} \hat{\eta}_{N x}=\hat{J}_{N} \frac{\delta\left(\operatorname{per}_{N} \tilde{H}_{n}\right)}{\delta \hat{\eta}_{N}}=\hat{M}_{N} \frac{\delta\left(\operatorname{per}_{N} \tilde{H}_{n-1}\right)}{\delta \hat{\eta}_{N}}
$$

where the Hamiltonian functionals $\operatorname{per}_{N} \tilde{H}_{n}$, the hereditary recursion operator $\hat{\Phi}_{N}$ and the Hamiltonian pair $\left\{\hat{J}_{N}, \hat{M}_{N}\right\}$ are given by

$$
\operatorname{per}_{N} \tilde{H}_{n}=\left.\frac{1}{N !} \frac{\partial^{N} \tilde{H}_{n}\left(\hat{u}_{N}\right)}{\partial \varepsilon^{N}}\right|_{\varepsilon=0}
$$




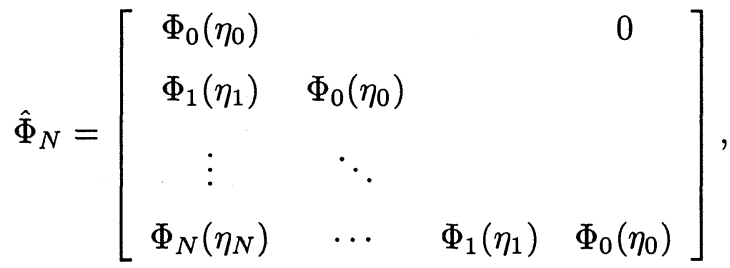

$$
\begin{aligned}
& \hat{J}_{N}=\left[\begin{array}{cccc}
0 & & & \partial_{x} \\
& & \partial_{x} & \\
& . & & \\
\partial_{x} & & & 0
\end{array}\right], \hat{M}_{N}=\left[\begin{array}{cccc}
0 & & & M_{0}\left(\eta_{0}\right) \\
& & M_{0}\left(\eta_{0}\right) & M_{1}\left(\eta_{1}\right) \\
& \therefore & . & \vdots \\
M_{0}\left(\eta_{0}\right) & M_{1}\left(\eta_{1}\right) & \ldots & M_{N}\left(\eta_{N}\right)
\end{array}\right] \text {, }
\end{aligned}
$$

with

$$
\begin{aligned}
& M_{i}=M_{i}\left(\eta_{i}\right)=\delta_{i 0} \partial_{x}^{3}+2\left(\partial_{x} \eta_{i}+\eta_{i} \partial_{x}\right), \\
& \Phi_{i}=\Phi_{i}\left(\eta_{i}\right)=\delta_{i 0} \partial_{x}^{2}+2\left(\partial_{x} \eta_{i} \partial_{x}^{-1}+\eta_{i}\right), \quad 0 \leq i \leq N
\end{aligned}
$$

Moreover they have infinitely many commuting symmetries $\left\{\hat{K}_{m N}\right\}_{m=0}^{\infty}$ and conserved densities $\left\{\hat{H}_{m N}\right\}_{m=0}^{\infty}$.

We list the first two standard perturbation systems of the KdV equation (3.5):

$$
\begin{aligned}
& \left\{\begin{array}{l}
\eta_{0 t}=\eta_{0 x x x}+6 \eta_{0} \eta_{0 x}, \\
\eta_{1 t}=\eta_{1 x x x}+6\left(\eta_{0} \eta_{1}\right)_{x}
\end{array}\right. \\
& \left\{\begin{array}{l}
\eta_{0 t}=\eta_{0 x x x}+6 \eta_{0} \eta_{0 x}, \\
\eta_{1 t}=\eta_{1 x x x}+6\left(\eta_{0} \eta_{1}\right)_{x}, \\
\eta_{2 t}=\eta_{2 x x x}+6 \eta_{1} \eta_{1 x}+6\left(\eta_{0} \eta_{2}\right)_{x}
\end{array}\right.
\end{aligned}
$$

The first-order perturbation system (3.13) has the following bi-Hamiltonian formulation

$\hat{\eta}_{1 t}=\left[\begin{array}{cc}0 & \partial_{x} \\ \partial_{x} & 0\end{array}\right] \frac{\delta\left(\operatorname{per}_{1} \tilde{H}_{1}\right)}{\delta \hat{\eta}_{1}}=\left[\begin{array}{cc}0 & \partial_{x}^{3}+2 \eta_{0 x}+4 \eta_{0} \partial_{x} \\ \partial_{x}^{3}+2 \eta_{0 x}+4 \eta_{0} \partial_{x} & 2 \eta_{1 x}+4 \eta_{1} \partial_{x}\end{array}\right] \frac{\delta\left(\operatorname{per}_{1} \tilde{H}_{0}\right)}{\delta \hat{\eta}_{1}}$ with $\hat{\eta}_{1}=\left(\eta_{0}, \eta_{1}\right)^{T}$ and the Hamiltonian functionals

$$
\begin{aligned}
\operatorname{per}_{1} \tilde{H}_{0} & =\int \hat{H}_{01} d x, \hat{H}_{01}=\eta_{0} \eta_{1}, \\
\operatorname{per}_{1} \tilde{H}_{1} & =\int \hat{H}_{11} d x, \hat{H}_{11}=\frac{1}{2}\left(\eta_{0} \eta_{1 x x}+\eta_{0 x x} \eta_{1}\right)+3 \eta_{0}^{2} \eta_{1} .
\end{aligned}
$$

The second-order perturbation system (3.14) has the following bi-Hamiltonian formulation

$$
\hat{\eta}_{2 t}=\hat{J}_{2} \frac{\delta\left(\operatorname{per}_{2} \tilde{H}_{1}\right)}{\delta \hat{\eta}_{2}}=\hat{M}_{2} \frac{\delta\left(\operatorname{per}_{2} \tilde{H}_{0}\right)}{\delta \hat{\eta}_{2}}, \hat{\eta}_{2}=\left(\eta_{0}, \eta_{1}, \eta_{2}\right)^{T}
$$

with the Hamiltonian functionals

$$
\operatorname{per}_{2} \tilde{H}_{0}=\int \hat{H}_{02} d x, \hat{H}_{02}=\eta_{0} \eta_{2}+\frac{1}{2} \eta_{1}^{2},
$$




$$
\operatorname{per}_{2} \tilde{H}_{1}=\int \hat{H}_{12} d x, \hat{H}_{12}=\frac{1}{2}\left(\eta_{0} \eta_{2 x x}+\eta_{1} \eta_{1 x x}+\eta_{0 x x} \eta_{2}\right)+3 \eta_{0} \eta_{1}^{2}+3 \eta_{0}^{2} \eta_{2}
$$

Another example that we want to show is the first-order standard perturbation system of the fifth order $\mathrm{KdV}$ equation $u_{t}=K_{2}(u)$ :

$$
\left\{\begin{aligned}
\eta_{0 t}=\eta_{0,5 x} & +10 \eta_{0} \eta_{0 x x x}+20 \eta_{0 x} \eta_{0 x x}+30 \eta_{0}^{2} \eta_{0 x} \\
\eta_{1 t}=\eta_{1,5 x} & +10 \eta_{0 x x x} \eta_{1}+10 \eta_{0} \eta_{1 x x x}+20 \eta_{0 x x} \eta_{1 x} \\
& +20 \eta_{0 x} \eta_{1 x x}+60 \eta_{0} \eta_{0 x} \eta_{1}+30 \eta_{0}^{2} \eta_{1 x}
\end{aligned}\right.
$$

where $\eta_{0,5 x}$ and $\eta_{1,5 x}$, as usual, stand for the fifth order derivatives of $\eta_{0}$ and $\eta_{1}$ with respect to $x$. It has the following bi-Hamiltonian formulation

$$
\hat{\eta}_{2 t}=\hat{J}_{1} \frac{\delta\left(\operatorname{per}_{1} \tilde{H}_{2}\right)}{\delta \hat{\eta}_{1}}=\hat{M}_{1} \frac{\delta\left(\operatorname{per}_{1} \tilde{H}_{1}\right)}{\delta \hat{\eta}_{1}}
$$

where the Hamiltonian function $\operatorname{per}_{1} \tilde{H}_{1}$ is given as before and the Hamiltonian function $\operatorname{per}_{1} \tilde{H}_{2}$, given as follows

$$
\begin{aligned}
\operatorname{per}_{1} \tilde{H}_{2}=\int\left(\frac{1}{2} \eta_{0 x x x x} \eta_{1}\right. & +\frac{1}{2} \eta_{0} \eta_{1 x x x x}+\frac{20}{3} \eta_{0} \eta_{0 x x} \eta_{1}+\frac{10}{3} \eta_{0}^{2} \eta_{1 x x} \\
& \left.+\frac{5}{3} \eta_{0 x}^{2} \eta_{1}+\frac{10}{3} \eta_{0} \eta_{0 x} \eta_{1 x}+\frac{40}{3} \eta_{0}^{3} \eta_{1}\right) d x
\end{aligned}
$$

3.2. Nonstandard perturbation systems. Secondly, let us choose a perturbed equation

$$
u_{t}=K^{\mathrm{per}}(u, \varepsilon)=K_{n}+\alpha \varepsilon K_{n}, \alpha=\text { const., } \alpha \neq 0,
$$

as an initial equation for each $n \geq 1$. This equation can be viewed as

$$
\begin{aligned}
u_{t}=K^{\mathrm{per}}(u, \varepsilon) & =J \frac{\delta\left(\tilde{H}_{n}+\alpha \varepsilon \tilde{H}_{n}\right)}{\delta u}=M \frac{\delta\left(\tilde{H}_{n-1}+\alpha \varepsilon \tilde{H}_{n-1}\right)}{\delta u} \\
& =(J+\alpha \varepsilon J) \frac{\delta \tilde{H}_{n}}{\delta u}=(M+\alpha \varepsilon M) \frac{\delta \tilde{H}_{n-1}}{\delta u}
\end{aligned}
$$

Therefore the corresponding perturbation systems also have quadruple Hamiltonian formulations. We focus on the first-order perturbation system under the single scale perturbation. It has the quadruple Hamiltonian formulation

$\hat{\eta}_{1 t}=\hat{J}_{1}^{(1)} \frac{\delta\left(\operatorname{per}_{1} \tilde{H}_{n}^{(1)}\right)}{\delta \hat{\eta}_{1}}=\hat{M}_{1}^{(1)} \frac{\delta\left(\operatorname{per}_{1} \tilde{H}_{n-1}^{(1)}\right)}{\delta \hat{\eta}_{1}}=\hat{J}_{1}^{(2)} \frac{\delta\left(\operatorname{per}_{1} \tilde{H}_{n}^{(2)}\right)}{\delta \hat{\eta}_{1}}=\hat{M}_{1}^{(2)} \frac{\delta\left(\operatorname{per}_{1} \tilde{H}_{n-1}^{(2)}\right)}{\delta \hat{\eta}_{1}}$,

namely,

$$
\begin{array}{r}
\hat{\eta}_{1 t}=\left[\begin{array}{ll}
0 & \partial \\
\partial & 0
\end{array}\right] \frac{\delta\left(\operatorname{per}_{1} \tilde{H}_{n}+\alpha \tilde{H}_{n}\left(\eta_{0}\right)\right)}{\delta \hat{\eta}_{1}}=\left[\begin{array}{cc}
0 & M_{0} \\
M_{0} & M_{1}
\end{array}\right] \frac{\delta\left(\operatorname{per}_{1} \tilde{H}_{n-1}+\alpha \tilde{H}_{n-1}\left(\eta_{0}\right)\right)}{\delta \hat{\eta}_{1}} \\
=\left[\begin{array}{cc}
0 & \partial \\
\partial & \alpha \partial
\end{array}\right] \frac{\delta\left(\operatorname{per}_{1} \tilde{H}_{n}\right)}{\delta \hat{\eta}_{1}}=\left[\begin{array}{cc}
0 & M_{0} \\
M_{0} & M_{1}+\alpha M_{0}
\end{array}\right] \frac{\delta\left(\operatorname{per}_{1} \tilde{H}_{n-1}\right)}{\delta \hat{\eta}_{1}}
\end{array}
$$


where the functionals $\operatorname{per}_{1} \tilde{H}_{n}, \operatorname{per}_{1} \tilde{H}_{n-1}$ and the operators $M_{i}$ are defined by (3.12) and (3.12), respectively. Since two Hamiltonian operators $\hat{J}_{1}^{(1)}$ and $\hat{J}_{1}^{(2)}$ are invertible, we can obtain five hereditary recursion operators for the equation (3.20):

$$
\begin{aligned}
& \hat{J}_{1}^{(2)}\left(\hat{J}_{1}^{(1)}\right)^{-1}=\left[\begin{array}{ll}
1 & 0 \\
\alpha & 1
\end{array}\right], \hat{J}_{1}^{(1)}\left(\hat{J}_{1}^{(2)}\right)^{-1}=\left[\begin{array}{cc}
1 & 0 \\
-\alpha & 1
\end{array}\right], \\
& \hat{M}_{1}^{(1)}\left(\hat{J}_{1}^{(2)}\right)^{-1}=\left[\begin{array}{cc}
\Phi_{0} & 0 \\
\Phi_{1}-\alpha \Phi_{0} & \Phi_{0}
\end{array}\right], \hat{M}_{1}^{(2)}\left(\hat{J}_{1}^{(1)}\right)^{-1}=\left[\begin{array}{cc}
\Phi_{0} & 0 \\
\Phi_{1}+\alpha \Phi_{0} & \Phi_{0}
\end{array}\right], \\
& \hat{M}_{1}^{(1)}\left(\hat{J}_{1}^{(1)}\right)^{-1}=\hat{M}_{1}^{(2)}\left(\hat{J}_{1}^{(2)}\right)^{-1}=\left[\begin{array}{cc}
\Phi_{0} & 0 \\
\Phi_{1} & \Phi_{0}
\end{array}\right],
\end{aligned}
$$

where the operators $\Phi_{i}$ are defined by (3.12). These operator structures suggest two classes of hereditary recursion operators for the equation (3.20)

$$
\hat{\Phi}_{1}^{(1)}(\beta)=\left[\begin{array}{ll}
\beta_{0} & 0 \\
\beta_{1} & \beta_{0}
\end{array}\right], \hat{\Phi}_{1}^{(2)}(\beta)=\left[\begin{array}{cc}
\beta_{0} \Phi_{0} & 0 \\
\beta_{0} \Phi_{1}+\beta_{1} \Phi_{0} & \beta_{0} \Phi_{0}
\end{array}\right],
$$

where $\beta=\left(\beta_{0}, \beta_{1}\right)^{T}$ with the $\beta_{i}$ being arbitrary constants. They are really hereditary operators and recursion operators for the equation (3.20), which can be verified by direct computation or by viewing them as the first-order perturbation operators of the initial operators $\beta_{0}+\beta_{1} \varepsilon$ and $\beta_{0} \Phi+\beta_{1} \varepsilon \Phi$. Therefore the integrable coupling (3.20) of the $n$-th order KdV equation $u_{t}=K_{n}(u)$ possesses two classes of hereditary recursion operators defined by (3.22). These two classes of operators have the property

$$
\Phi_{1}^{(1)}(\beta) \Phi_{1}^{(2)}(\gamma)=\Phi_{1}^{(2)}(\beta) \Phi_{1}^{(1)}(\gamma)=\left[\begin{array}{cc}
\beta_{0} \gamma_{0} \Phi_{0} & 0 \\
\beta_{0} \gamma_{0} \Phi_{1}+\left(\beta_{0} \gamma_{1}+\beta_{1} \gamma_{0}\right) \Phi_{0} & \beta_{0} \gamma_{0} \Phi_{0}
\end{array}\right],
$$

for any two constant vectors $\beta=\left(\beta_{0}, \beta_{1}\right)^{T}$ and $\gamma=\left(\gamma_{0}, \gamma_{1}\right)^{T}$, which also shows that their product can not constitute completely new recursion operators.

We also start from the perturbed KdV type equation

$$
u_{t}=K^{\text {per }}(u, \varepsilon)=K_{n}+\alpha \varepsilon^{j} K_{i_{j}}, \alpha=\text { const., } \alpha \neq 0,
$$

where $i_{j}$ is a natural number. Let us illustrate the idea of construction by the following specific example

$$
u_{t}=K^{\text {per }}(u, \varepsilon)=K_{n}+\alpha \varepsilon^{2} K_{n+1}(n \geq 1),
$$

which can be viewed as a tri-Hamiltonian system:

$$
u_{t}=K^{\text {per }}=J \frac{\delta\left(\tilde{H}_{n}+\alpha \varepsilon^{2} \tilde{H}_{n+1}\right)}{\delta u}=\left(J+\alpha \varepsilon^{2} M\right) \frac{\delta \tilde{H}_{n}}{\delta u}=M \frac{\delta\left(\tilde{H}_{n-1}+\alpha \varepsilon^{2} \tilde{H}_{n}\right)}{\delta u} .
$$

Therefore, according to Theorem 2.9, the second-order perturbation system of the the perturbed system (3.25)

$$
\left\{\begin{array}{l}
\eta_{0 t}=K_{n}\left(\eta_{0}\right) \\
\eta_{1 t}=K_{n}^{\prime}\left(\eta_{0}\right)\left[\eta_{1}\right] \\
\eta_{2 t}=\left.\frac{1}{2} \frac{\partial^{2} K_{n}\left(\hat{u}_{2}\right)}{\partial \varepsilon^{2}}\right|_{\varepsilon=0}+\alpha K_{n+1}\left(\eta_{0}\right)
\end{array}\right.
$$


possesses the following tri-Hamiltonian formulation

$$
\hat{\eta}_{2 t}=\hat{J}_{2}^{(1)} \frac{\delta \tilde{H}_{n}^{(1)}}{\delta \hat{\eta}_{2}}=\hat{J}_{2}^{(2)} \frac{\delta \tilde{H}_{n}^{(2)}}{\delta \hat{\eta}_{2}}=\hat{J}_{2}^{(3)} \frac{\delta \tilde{H}_{n}^{(3)}}{\delta \hat{\eta}_{2}}, \hat{\eta}_{2}=\left(\eta_{0}, \eta_{1}, \eta_{2}\right)^{T}
$$

with a triple of Hamiltonian operators

$$
\hat{J}_{2}^{(1)}=\left[\begin{array}{ccc}
0 & 0 & \partial_{x} \\
0 & \partial_{x} & 0 \\
\partial_{x} & 0 & 0
\end{array}\right], \hat{J}_{2}^{(2)}=\left[\begin{array}{ccc}
0 & 0 & \partial_{x} \\
0 & \partial_{x} & 0 \\
\partial_{x} & 0 & \alpha M_{0}
\end{array}\right], \hat{J}_{2}^{(3)}=\left[\begin{array}{ccc}
0 & 0 & M_{0} \\
0 & M_{0} & M_{1} \\
M_{0} & M_{1} & M_{2}
\end{array}\right]
$$

and the corresponding three Hamiltonian functionals

$$
\left\{\begin{array}{l}
\tilde{H}_{n}^{(1)}\left(\hat{\eta}_{2}\right)=\left(\operatorname{per}_{2} \tilde{H}_{n}\right)\left(\hat{\eta}_{2}\right)+\alpha \tilde{H}_{n+1}\left(\eta_{0}\right) \\
\tilde{H}_{n}^{(2)}\left(\hat{\eta}_{2}\right)=\left(\operatorname{per}_{2} \tilde{H}_{n}\right)\left(\hat{\eta}_{2}\right) \\
\tilde{H}_{n}^{(3)}\left(\hat{\eta}_{2}\right)=\left(\operatorname{per}_{2} \tilde{H}_{n-1}\right)\left(\hat{\eta}_{2}\right)+\alpha \tilde{H}_{n}\left(\eta_{0}\right)
\end{array}\right.
$$

Similarly, the perturbation system (3.27) has also two classes of hereditary recursion operators:

$$
\begin{aligned}
\hat{\Phi}_{2}^{(1)}(\beta) & =\left[\begin{array}{lll}
\beta_{0} & 0 & 0 \\
\beta_{1} & \beta_{0} & \\
\beta_{2} & \beta_{1} & \beta_{0}
\end{array}\right], \\
\hat{\Phi}_{2}^{(2)}(\beta) & =\left[\begin{array}{ccc}
\beta_{0} \Phi_{0} & 0 & 0 \\
\beta_{0} \Phi_{1}+\beta_{1} \Phi_{0} & \beta_{0} \Phi_{0} & \\
\beta_{0} \Phi_{2}+\beta_{1} \Phi_{1}+\beta_{2} \Phi_{0} & \beta_{0} \Phi_{1}+\beta_{1} \Phi_{0} & \beta_{0} \Phi_{0}
\end{array}\right],
\end{aligned}
$$

where the operators $\Phi_{i}$ are defined by (3.12) and $\beta=\left(\beta_{0}, \beta_{1}, \beta_{2}\right)^{T}$ is a constant vector.

Let us fix $n=1$ and then the system (3.27) gives an integrable coupling of the $\mathrm{KdV}$ equation (3.5), which possesses the following tri-Hamiltonian formulation

$$
\hat{\eta}_{2 t}=\hat{J}_{2}^{(1)} \frac{\delta \tilde{H}_{1}^{(1)}}{\delta \hat{\eta}_{2}}=\hat{J}_{2}^{(2)} \frac{\delta \tilde{H}_{1}^{(2)}}{\delta \hat{\eta}_{2}}=\hat{J}_{2}^{(3)} \frac{\delta \tilde{H}_{1}^{(3)}}{\delta \hat{\eta}_{2}}
$$

with three Hamiltonian functionals

$$
\left\{\begin{aligned}
\tilde{H}_{1}^{(1)}\left(\hat{\eta}_{2}\right)= & \int\left[\frac{1}{2}\left(\eta_{0} \eta_{2 x x}+\eta_{1} \eta_{1 x x}+\eta_{0 x x} \eta_{2}\right)+3 \eta_{0} \eta_{1}^{2}+3 \eta_{0}^{2} \eta_{2}\right. \\
& \left.+\alpha\left(\frac{1}{2} \eta_{0} \eta_{0 x x x x}+\frac{10}{3} \eta_{0}^{2} \eta_{0 x x}+\frac{5}{3} \eta_{0} \eta_{0 x}^{2}+\frac{10}{3} \eta_{0}^{4}\right)\right] d x \\
\tilde{H}_{1}^{(2)}\left(\hat{\eta}_{2}\right)= & \int\left[\eta_{0} \eta_{2}+\frac{1}{2} \eta_{1}^{2}+\alpha\left(\frac{1}{2} \eta_{0} \eta_{0 x x}+\eta_{0}^{3}\right)\right] d x \\
\tilde{H}_{1}^{(3)}\left(\hat{\eta}_{2}\right)= & \int\left[\frac{1}{2}\left(\eta_{0} \eta_{2 x x}+\eta_{1} \eta_{1 x x}+\eta_{0 x x} \eta_{2}\right)+3 \eta_{0} \eta_{1}^{2}+3 \eta_{0}^{2} \eta_{2}\right] d x
\end{aligned}\right.
$$

In order to distinguish the standard perturbation systems defined by (3.10), the integrable couplings of the $n$-th order $\mathrm{KdV}$ equation $u_{t}=K_{n}$, defined by (3.20) and (3.27), are called the non-standard perturbation systems. Interestingly, each of these systems has both a local multi-Hamiltonian formulation and two classes of hereditary recursion operators. 
3.3. $2+1$ dimensional integrable couplings. Thirdly, let us consider a case of bi-scale perturbations (2.41), i.e.,

$$
\hat{u}_{N}=\sum_{i=0}^{N} \varepsilon^{i} \eta_{i}, \eta_{i}=\eta_{i}(x, y, t), y=\varepsilon x
$$

In order to present explicit results for integrable couplings, we take the KdV equation (3.5) as an illustrative example, due to its simplicity. We recall that the KdV equation (3.5) has the bi-Hamiltonian formulation (3.6) and the Lax pair (3.8).

Let us introduce the bi-scale perturbation series above into the KdV equation (3.5) and equate powers of $\varepsilon$. As an $N$-th order approximation, we obtain a $2+1$ dimensional perturbation systems of evolution equations

$$
\left\{\begin{aligned}
\eta_{0 t_{1}}= & \eta_{0 x x x}+6 \eta_{0} \eta_{0 x} \\
\eta_{1 t_{1}} & =\eta_{1 x x x}+3 \eta_{0 x x y}+6\left(\eta_{0} \eta_{1}\right)_{x}+6 \eta_{0} \eta_{0 y} \\
\eta_{2 t_{1}} & =\eta_{2 x x x}+3 \eta_{1 x x y}+3 \eta_{0 x y y}+6\left(\eta_{0} \eta_{2}\right)_{x}+6 \eta_{1} \eta_{1 x}+6\left(\eta_{0} \eta_{1}\right)_{y} \\
\eta_{j t_{1}}= & \eta_{j x x x}+3 \eta_{j-1, x x y}+3 \eta_{j-2, x y y}+\eta_{j-3, y y y} \\
& +6\left(\sum_{i=0}^{j} \eta_{i} \eta_{j-i, x}+\sum_{i=0}^{j-1} \eta_{i} \eta_{j-i-1, y}\right), 3 \leq j \leq N
\end{aligned}\right.
$$

This system has been already presented in [20]. It follows from our general theory that it gives an integrable coupling of the $\mathrm{KdV}$ equation (3.5).

In what follows, we would like to propose a bi-Hamiltonian formulation and the consequent hereditary recursion operator for the system (3.34). To the end, we first need to compute a perturbation Hamiltonian pair by Theorem 2.3:

$(3.35) \hat{J}_{N}=\left[\begin{array}{ccccc}0 & & & & \partial_{x} \\ & & & \partial_{x} & \partial_{y} \\ & & \partial_{x} & \partial_{y} & 0 \\ & \therefore & \therefore & \therefore & \vdots \\ \partial_{x} & \partial_{y} & 0 & \cdots & 0\end{array}\right]$

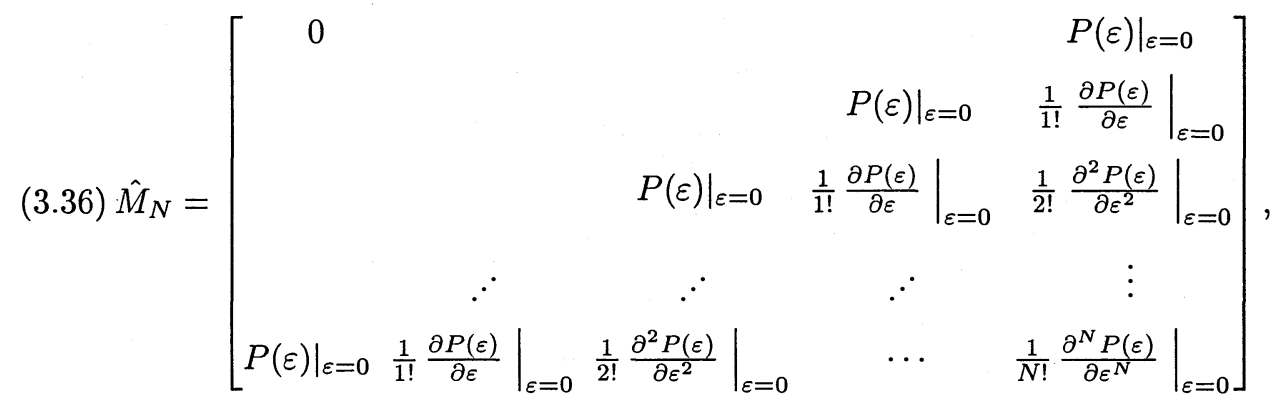

where the differential operator $P(\varepsilon)$ represents

$$
P(\varepsilon)=\left(\partial_{x}+\varepsilon \partial_{y}\right)^{3}+2\left[\left(\partial_{x}+\varepsilon \partial_{y}\right) \hat{u}_{N}+\hat{u}_{N}\left(\partial_{x}+\varepsilon \partial_{y}\right)\right]
$$

The explicit expressions for various derivatives of $P(\varepsilon)$ with respect to $\varepsilon$ can be ob- 
tained as follows:

$$
\left\{\begin{array}{l}
\left.P(\varepsilon)\right|_{\varepsilon=0}=\partial_{x}^{3}+2\left(\partial_{x} \eta_{0}+\eta_{0} \partial_{x}\right), \\
\left.\frac{1}{1 !} \frac{\partial P(\varepsilon)}{\partial \varepsilon}\right|_{\varepsilon=0}=3 \partial_{x}^{2} \partial_{y}+2\left(\partial_{x} \eta_{1}+\eta_{1} \partial_{x}\right)+2\left(\partial_{y} \eta_{0}+\eta_{0} \partial_{y}\right), \\
\left.\frac{1}{2 !} \frac{\partial^{2} P(\varepsilon)}{\partial \varepsilon^{2}}\right|_{\varepsilon=0}=3 \partial_{x} \partial_{y}^{2}+2\left(\partial_{x} \eta_{2}+\eta_{2} \partial_{x}\right)+2\left(\partial_{y} \eta_{1}+\eta_{1} \partial_{y}\right), \\
\left.\frac{1}{3 !} \frac{\partial^{3} P(\varepsilon)}{\partial \varepsilon^{3}}\right|_{\varepsilon=0}=\partial_{y}^{3}+2\left(\partial_{x} \eta_{3}+\eta_{3} \partial_{x}\right)+2\left(\partial_{y} \eta_{2}+\eta_{2} \partial_{y}\right), \\
\left.\frac{1}{i !} \frac{\partial^{i} P(\varepsilon)}{\partial \varepsilon^{i}}\right|_{\varepsilon=0}=2\left(\partial_{x} \eta_{i}+\eta_{i} \partial_{x}\right)+2\left(\partial_{y} \eta_{i-1}+\eta_{i-1} \partial_{y}\right), 4 \leq i \leq N,
\end{array}\right.
$$

which gives rise to an explicit expression for the Hamiltonian operator $\hat{M}_{N}$. Secondly, we need to compute the Hamiltonian functionals for the system (3.34). Note that $\partial_{x} \rightarrow \partial_{x}+\varepsilon \partial_{y}$, and thus, under the perturbation (2.41), we have

$$
u_{x x} \rightarrow \sum_{i=0}^{N} \varepsilon^{i}\left(\eta_{i x x}+2 \varepsilon \eta_{i x y}+\varepsilon^{2} \eta_{i y y}\right) .
$$

Further, by Theorem 2.9, we obtain two perturbation Hamiltonian functionals:

$$
\begin{aligned}
\operatorname{per}_{N} \tilde{H}_{0}= & \left.\iint \frac{1}{N !} \frac{\partial^{N} H_{0}\left(\hat{u}_{N}\right)}{\partial \varepsilon^{N}}\right|_{\varepsilon=0} d x d y=\iint \frac{1}{2} \sum_{i=0}^{N} \eta_{i} \eta_{N-i} d x d y \\
\operatorname{per}_{N} \tilde{H}_{1}= & \left.\iint \frac{1}{N !} \frac{\partial^{N} H_{1}\left(\hat{u}_{N}\right)}{\partial \varepsilon^{N}}\right|_{\varepsilon=0} d x d y=\iint\left[\frac{1}{2} \sum_{i+j=N} \eta_{i} \eta_{j x x}\right. \\
& \left.+\sum_{i+j=N-1} \eta_{i} \eta_{j x y}+\frac{1}{2} \sum_{i+j=N-2} \eta_{i} \eta_{j y y}+\sum_{i+j+k=N} \eta_{i} \eta_{j} \eta_{k}\right] d x d y
\end{aligned}
$$

Now the following bi-Hamiltonian formulation for the system (3.34) becomes clear:

$$
\hat{\eta}_{N t}=\hat{J}_{N} \frac{\delta\left(\operatorname{per}_{N} \tilde{H}_{1}\right)}{\delta \hat{\eta}_{N}}=\hat{M}_{N} \frac{\delta\left(\operatorname{per}_{N} \tilde{H}_{0}\right)}{\delta \hat{\eta}_{N}}
$$

where $\hat{J}_{N}, \hat{M}_{N}, \operatorname{per}_{N} \tilde{H}_{0}$ and $\operatorname{per}_{N} \tilde{H}_{1}$ are defined by (3.35), (3.36), (3.38) and (3.39), respectively. It should be realized that the $2+1$ dimensional bi-Hamiltonian system (3.40) is local, because the Hamiltonian pair $\left\{\hat{J}_{N}, \hat{M}_{N}\right\}$ involves only the differential operators $\partial_{x}$ and $\partial_{y}$.

Theorem 2.5 guarantees the existence of a hereditary recursion operator for the system (3.34). It is of interest to get its explicit expression. Note that the first Hamiltonian operator $\hat{J}_{N}$ has an invertible operator

$$
\left(\hat{J}_{N}\right)^{-1}=\left[\begin{array}{cccc}
P_{N} & P_{N-1} & \cdots & P_{0} \\
P_{N-1} & & \therefore & \\
\vdots & \therefore & & \\
P_{0} & & & 0
\end{array}\right]
$$

where the operators $P_{i}$ are defined by

$$
\stackrel{3.42)}{P_{0}}=\partial_{x}^{-1}, P_{1}=-\partial_{x}^{-2} \partial_{y}, \cdots, P_{i}=(-1)^{i} \partial_{x}^{-i-1} \partial_{y}^{i}, \cdots, P_{N}=(-1)^{N} \partial_{x}^{-N-1} \partial_{y}^{N} .
$$


Therefore, the corresponding hereditary recursion operator is determined by $\hat{\Phi}_{N}=$ $\hat{M}_{N} \hat{J}_{N}^{-1}$, but it can also be computed directly by Theorem 2.2 :

$$
\hat{\Phi}_{N}=\left[\begin{array}{cccc}
\left.\Phi\left(\hat{u}_{N}\right)\right|_{\varepsilon=0} & & & 0 \\
\left.\frac{1}{1 !} \frac{\partial \Phi\left(\hat{u}_{N}\right)}{\partial \varepsilon}\right|_{\varepsilon=0} & \left.\Phi\left(\hat{u}_{N}\right)\right|_{\varepsilon=0} & & \\
\vdots & \ddots & \ddots & \\
\left.\frac{1}{N !} \frac{\partial^{N} \Phi\left(\hat{u}_{N}\right)}{\partial \varepsilon^{N}}\right|_{\varepsilon=0} & \cdots & \left.\frac{1}{1 !} \frac{\partial \Phi\left(\hat{u}_{N}\right)}{\partial \varepsilon}\right|_{\varepsilon=0} & \left.\Phi\left(\hat{u}_{N}\right)\right|_{\varepsilon=0}
\end{array}\right] .
$$

Here the operator $\Phi\left(\hat{u}_{N}\right)$ is defined by

$$
\Phi\left(\hat{u}_{N}\right)=\left(\partial_{x}+\varepsilon \partial_{y}\right)^{2}+2\left(\hat{u}_{N x}+\varepsilon \hat{u}_{N y}\right)\left(\partial_{x}+\varepsilon \partial_{y}\right)^{-1}+4 \hat{u}_{N},
$$

and thus its $N+1$ derivatives with respect to $\varepsilon$ are found to be

$$
\left\{\begin{array}{l}
\left.\Phi\left(\hat{u}_{N}\right)\right|_{\varepsilon=0}=\partial_{x}^{2}+2 \eta_{0 x} \partial_{x}^{-1}+4 \eta_{0}, \\
\left.\frac{1}{1 !} \frac{\partial \Phi\left(\hat{u}_{N}\right)}{\partial \varepsilon}\right|_{\varepsilon=0}=2 \partial_{x} \partial_{y}+2\left(\eta_{1 x}+\eta_{0 y}\right) \partial_{x}^{-1}-2 \eta_{0 x} \partial_{x}^{-2} \partial_{y}+4 \eta_{1}, \\
\left.\frac{1}{2 !} \frac{\partial^{2} \Phi\left(\hat{u}_{N}\right)}{\partial \varepsilon^{2}}\right|_{\varepsilon=0}=\partial_{y}^{2}+2\left(\eta_{2 x}+\eta_{1 y}\right) \partial_{x}^{-1}-2\left(\eta_{1 x}+\eta_{0 y}\right) \partial_{x}^{-2} \partial_{y}+2 \eta_{0 x} \partial_{x}^{-3} \partial_{y}^{2}+4 \eta_{2}, \\
\left.\frac{1}{k !} \frac{\partial^{k} \Phi\left(\hat{u}_{N}\right)}{\partial \varepsilon^{k}}\right|_{\varepsilon=0}=\sum_{i+j=k}(-1)^{j}\left(2 \eta_{i x}+\eta_{i-1, y}\right) \partial_{x}^{-j-1} \partial_{y}^{j}+4 \eta_{k}, 3 \leq k \leq N,
\end{array}\right.
$$

where we accept $\eta_{-1}=0$.

Let us now show the corresponding zero curvature representation for the $2+1$ dimensional perturbation system (3.34). By Theorem 2.8 or (2.54), the zero curvature representation for the system (3.34) can be given by

$$
\hat{U}_{N t}-\hat{V}_{N x}-\Pi \hat{V}_{N y}+\left[\hat{U}_{N}, \hat{V}_{N}\right]=0,
$$

where three matrices $\Pi, \hat{U}_{N}$ and $\hat{V}_{N}$ read as

$$
\begin{aligned}
& \Pi=\left[\begin{array}{cc}
0 & 0 \\
I_{2 N} & 0
\end{array}\right]_{2(N+1) \times 2(N+1)}, I_{2 N}=\operatorname{diag}(\underbrace{I_{2}, \cdots, I_{2}}_{N})=\operatorname{diag}(\underbrace{1, \cdots, 1}_{2 N}), \\
& \hat{U}_{N}=\left[\begin{array}{cccc}
U_{0} & & & 0 \\
U_{1} & U_{0} & & \\
\vdots & \ddots & \ddots & \\
U_{N} & \cdots & U_{1} & U_{0}
\end{array}\right], \hat{V}_{N}=\left[\begin{array}{cccc}
V_{0} & & & 0 \\
V_{1} & V_{0} & & \\
\vdots & \ddots & \ddots & \\
V_{N} & \cdots & V_{1} & V_{0}
\end{array}\right] \text {, }
\end{aligned}
$$

with the $U_{i}, V_{i}$ being determined by

$$
\begin{aligned}
& U_{i}=\left.\frac{1}{i !} \frac{\partial^{i} U\left(\hat{u}_{N}, \hat{\lambda}_{N}\right)}{\partial \varepsilon^{i}}\right|_{\varepsilon=0}=\left[\begin{array}{cc}
0 & -\eta_{i}-\mu_{i} \\
\delta_{i 0} & 0
\end{array}\right], 0 \leq i \leq N \\
& V_{i}=\left.\frac{1}{i !} \frac{\partial^{i} V\left(\hat{u}_{N}, \hat{\lambda}_{N}\right)}{\partial \varepsilon^{i}}\right|_{\varepsilon=0}=\left[\begin{array}{cc}
\eta_{i x}+\eta_{i-1, y} & Q_{i} \\
2 \eta_{i}-4 \mu_{i} & -\eta_{i x}-\eta_{i-1, y}
\end{array}\right], 0 \leq i \leq N
\end{aligned}
$$




$$
Q_{i}=-\eta_{i x x}-2 \eta_{i-1, x y}-\eta_{i-2, y y}-2 \sum_{k+l=i}\left(\eta_{k} \eta_{l}-\mu_{k} \eta_{l}-2 \mu_{k} \mu_{l}\right), 0 \leq i \leq N
$$

where we accept that $\eta_{-1}=\eta_{-2}=0$, and $U, V$ are defined by (3.8). Of course, we require the condition (2.42) on the involved spectral parameters $\mu_{i}, 0 \leq i \leq N$, in order to guarantee the equivalence between the system (3.34) and the zero curvature equation (3.45).

In particular, the first-order bi-scale perturbation system

$$
\left\{\begin{array}{l}
\eta_{0 t}=\eta_{0 x x x}+6 \eta_{0} \eta_{0 x} \\
\eta_{1 t}=\eta_{1 x x x}+3 \eta_{0 x x y}+6\left(\eta_{0} \eta_{1}\right)_{x}+6 \eta_{0} \eta_{0 y}
\end{array}\right.
$$

has a local $2+1$ dimensional bi-Hamiltonian formulation

$$
\begin{aligned}
& \hat{\eta}_{1 t}=\hat{J}_{1} \frac{\delta\left(\operatorname{per}_{1} \tilde{H}_{1}\right)}{\delta \hat{\eta}_{1}}=\hat{M}_{1} \frac{\delta\left(\operatorname{per}_{1} \tilde{H}_{0}\right)}{\delta \hat{\eta}_{1}}, \hat{\eta}_{1}=\left[\begin{array}{c}
\eta_{0} \\
\eta_{1}
\end{array}\right], \hat{J}_{1}=\left[\begin{array}{cc}
0 & \partial_{x} \\
\partial_{x} & \partial_{y}
\end{array}\right], \\
& \hat{M}_{1}=\left[\begin{array}{cc}
0 & \partial_{x}^{3}+2 \eta_{0 x}+4 \eta_{0} \partial_{x} \\
\partial_{x}^{3}+2 \eta_{0 x}+4 \eta_{0} \partial_{x} & 3 \partial_{x}^{2} \partial_{y}+2 \eta_{1 x}+2 \eta_{0 y}+4 \eta_{1} \partial_{x}+4 \eta_{0} \partial_{y}
\end{array}\right], \\
& \operatorname{per}_{1} \tilde{H}_{0}=\iint \eta_{0} \eta_{1} d x d y, \operatorname{per}_{1} \tilde{H}_{1}=\iint\left(\frac{1}{2} \eta_{0} \eta_{1 x x}+\eta_{0} \eta_{0 x y}+\frac{1}{2} \eta_{1} \eta_{0 x x}+3 \eta_{0}^{2} \eta_{1}\right) d x d y .
\end{aligned}
$$

Here the extended variables $\eta_{0}(x, y, t)$ and $\eta_{1}(x, y, t)$ are taken as a potential vector $\hat{\eta}_{1}$. Moreover the above Hamiltonian pair yields a hereditary recursion operator in $2+1$ dimensions

$$
\hat{\Phi}_{1}\left(\hat{\eta}_{1}\right)=\left[\begin{array}{cc}
\partial_{x}^{2}+2 \eta_{0 x} \partial_{x}^{-1}+4 \eta_{0} & 0 \\
2 \partial_{x} \partial_{y}-2 \eta_{0 x} \partial_{x}^{-2} \partial_{y}+2\left(\eta_{1 x}+\eta_{0 y}\right) \partial_{x}^{-1}+4 \eta_{1} & \partial_{x}^{2}+2 \eta_{0 x} \partial_{x}^{-1}+4 \eta_{0}
\end{array}\right] .
$$

The system (3.51) was furnished in [20], its Painlevé property and zero curvature representation were discussed by Sakovich [21], and its localized soliton-like solutions were form? in [22]. All these properties show that the system (3.51) is a good example of typical soliton equations in $2+1$ dimensions.

4. Concluding remarks. We have developed a theory for constructing integrable couplings of soliton equations by perturbations. The symmetry problem is viewed as a special case of integrable couplings. The general structures of hereditary recursion operators, Hamiltonian operators, symplectic operators, Hamiltonian formulations etc. have been established under the multi-scale perturbations. The perturbation systems have richer structures of Lax representations and zero curvature representations than the original systems. For example, in the higher dimensional cases, the involved spectral parameters $\mu_{i}, 0 \leq i \leq N$, may vary with respect to the spatial variables, but they need to satisfy some conditions, for example,

$$
\mu_{0 x}=0, \mu_{i x}+\mu_{i-1, y}=0,1 \leq i \leq N
$$

in the $2+1$ dimensional case of the perturbation

$$
\hat{u}_{n}=\sum_{i=0}^{N} \varepsilon^{i} \eta_{i}(x, y, t)=\sum_{i=0}^{N} \varepsilon^{i} \eta_{i}(x, \varepsilon x, t), x \in \mathbb{R} .
$$


The resulting theory has been applied to the $\mathrm{KdV}$ soliton hierarchy and thus various integrable couplings are presented for each soliton equation in the KdV hierarchy. The obtained integrable couplings of the original $\mathrm{KdV}$ equations have infinitely many commuting symmetries and conserved densities. Linear combinations of the KdV hierarchy containing a small perturbation parameter may yield much more interesting integrable couplings. For example, the KdV type systems of soliton equations possessing both multi-Hamiltonian formulations and two classes of hereditary recursion operators have been presented and what's more, local $2+1$ dimensional bi-Hamiltonian systems of the $\mathrm{KdV}$ type with hereditary structures have also been constructed.

Our success in extending the standard perturbation cases to the non-standard cases and the higher dimensional cases are based on the following two simple ideas. First, we chose the perturbed systems as initial systems to generate integrable couplings for given integrable systems. The method of construction is similar to that in [5]. Only a slight difference is that new initial systems themselves involve a small perturbation parameter, but importantly, such initial perturbed systems take effect in getting new integrable couplings. In particular, our result showed that the following non-standard perturbation system

$$
\left\{\begin{array}{l}
u_{t}=K(u) \\
v_{t}=K^{\prime}(u)[v]+K(u)
\end{array}\right.
$$

keeps complete integrability. Therefore, this also provides us with an extension of integrable systems. Secondly, we took the multi-scale perturbations, by which higher dimensional integrable couplings can be presented. Indeed, the multi-scale perturbations enlarge the spatial dimensions and keeps complete integrability of the system under study. A concrete example of integrable couplings resulted from the multi-scale perturbations is the following system

$$
\left\{\begin{array}{l}
\eta_{0 t}=\eta_{0 x x x}+6 \eta_{0} \eta_{0 x} \\
\eta_{1 t}=\eta_{1 x x x}+3 \eta_{0 x x y}+6\left(\eta_{0} \eta_{1}\right)_{x}+6 \eta_{0} \eta_{0 y}
\end{array}\right.
$$

which has been proved to be a local bi-Hamiltonian system.

A kind of reduction of the standard multi-scale perturbations defined by (2.1) may be taken, which can be generally represented as

$$
\hat{u}_{N}=\sum_{j=0}^{N} \varepsilon^{i_{j}} \eta_{j}, \eta_{i}=\eta_{i}\left(y_{0}, y_{1}, y_{2}, \cdots, y_{r}, t\right), y_{j}=\varepsilon^{i_{j}^{\prime}} x, t \in \mathbb{R}, x \in \mathbb{R}^{p}, 0 \leq i \leq N
$$

where the $i_{j}, i_{j}^{\prime}$ can be any two finite sets of natural numbers. This kind of perturbations can be generated from the standard perturbations (2.1), if some dependent variables $\eta_{i}$ are chosen to be zero and the other dependent variables are assumed to be independent of some dependent variables $y_{i}$. They yield more specific integrable couplings. There is another interesting problem related integrable couplings. Could one reduce the spatial dimensions of a given integrable system while formulating integrable couplings? If the answer is yes, it is of interest to find some ways to construct such kind of integrable couplings, i.e., to hunt for the second part $S(u, v)$ with $v$ being less dimensional than $u$ to constitute integrable systems with the original system $u_{t}=K(u)$.

There exist some important works to deal with asymptotic analysis and asymptotic integrability $[23,24,25,26]$, to which the study of the perturbation systems 
may be helpful. It is also worthy mentioning that our $2+1$ dimensional hereditary recursion operators, for example, the operators defined by (3.43) and (3.44), are of the form described only by independent variables involved. Thus they are a supplement to a theory of recursion operators in $2+1$ dimensions discussed by Zakharov and Konopelchenko [27], and a theory of the extended recursion operators in $2+1$ dimensions including additional independent variables, introduced by Santini and Fokas [28] and Fokas and Santini [29]. The other properties such as Bäcklund transformations, bilinear forms and soliton solutions might be found for the resulting perturbation systems. A remarkable Miura transformation [30] might also be introduced for the $2+1$ dimensional perturbation systems (3.34), which will lead to new $2+1$ dimensional integrable systems of the MKdV type. All these problems will be analyzed in a further publication.

ACKNowledgments. The author would like to thank the City University of Hong Kong (Project nos. 7000945 and 7001041) and the Research Grants Council of Hong Kong (Project nos. 9040395 and 9040466) for financial support.

\section{REFERENCES}

[1] B. FUChSSTEINER, Coupling of completely integrable systems: the perturbation bundle, in Applications of Analytic and Geometric Methods to Nonlinear Differential Equations, P. A. Clarkson, ed., Kluwer, Dordrecht, 1993, pp. 125-138.

[2] W. X. MA, Lax representations and Lax operator algebras of isospectral and nonisospectral hierarchies of evolution equations, J. Math. Phys., 33 (1992), pp. 2464-2476.

[3] W. X. MA, An approach for constructing nonisospectral hierarchies of evolution equations, J. Phys. A: Math. Gen., 25 (1992), pp. L719-L726; A simple scheme for generating nonisospectral flows from zero curvature representation, Phys. Lett. A, 179 (1993), pp. 179-185.

[4] M. LAKSHMANAN AND K. M. TAMIZHMANI, Lie-Bäcklund symmetries of certain nonlinear evolution equations under perturbation around solutions, J. Math. Phys., 26 (1985), pp. 11891200.

[5] W. X. MA AND B. Fuchssteiner, Integrable theory of the perturbation equations, Chaos, Solitons \& Fractals, 7 (1996), pp. 1227-1250.

[6] W. X. MA, A bi-Hamiltonian formulation for triangular systems by perturbations, preprint (1998).

[7] B. Fuchssteiner AND A. S. FoKas, Symplectic structures, their Bäcklund transformations and hereditary symmetries, Physica D, 4 (1981), pp. 47-66.

[8] W. OEvel, Dirac constraints in field theory: lifts of Hamiltonian systems to the cotangent bundle, J. Math. Phys., 29 (1988), pp. 210-219.

[9] B. FUCHSSTEINER, Application of hereditary symmetries to nonlinear evolution equations, Nonlinear Analysis TMA, 3 (1979), pp. 849-862.

[10] A. S. Fokas, Symmetries and integrability, Studies in Appl. Math., 77 (1987), pp. 253-299.

[11] C. H. Gu (ed.), Soliton Theory and Its Applications, Springer-Verlag, Berlin, 1995.

[12] P. J. Olver, Applications of Lie Groups to Differential Equations, Springer-Verlag, New York, 1986.

[13] I. M. Gelfand AND I. YA. DoRfman, Hamiltonian operators and algebraic structures related to them, Func. Anal. Appl., 13 (1979), pp. 248-262.

[14] I. YA. DoRfman, Dirac Structures and Integrability of Nonlinear Evolution Equations, John Wiley \& Sons, Chichester, 1993.

[15] F. MAGRI, A simple model of the integrable Hamiltonian equation, J. Math. Phys., 19 (1978), pp. 1156-1162.

[16] W. X. MA, The algebraic structures of isospectral Lax operators and applications to integrable equations, J. Phys. A: Math. Gen., 25 (1992), pp. 5329-5343.

[17] R. M. MiURA, The Korteweg-de Vries equation: a survey of results, SIAM Rev., 18 (1976), pp. $412-459$.

[18] M. J. Ablowitz AND H. Segur, Solitons and the Inverse Scattering Transform, SIAM, Philadelphia, 1981.

[19] K. M. Tamizhmani and M. Lakshmanan, Complete integrability of the Korteweg-de Vries equation under perturbation around its solution: Lie-Bäcklund symmetry approach, J. 
Phys. A: Math. Gen., 16 (1983), pp. 3773-3782.

[20] W. X. MA AND B. FUCHSSTEINER, The bi-Hamiltonian structure of the perturbation equations of the KdV hierarchy, Phys. Lett. A, 213 (1996), pp. 49-55.

[21] S. YU. SAKOVICH, On integrability of a $(2+1)$-dimensional perturbed KdV equation, J. Nonlinear Math. Phys., 5 (1998), pp. 230-233.

[22] Z. X. ZHOU AND W. X. MA, Darboux transformations for a $2+1$ dimensional matrix GelfandDickey system, preprint (1998).

[23] R. Wong, Asymptotic Approximations of Integrals, Computer Science and Scientific Computing, Academic Press, Boston, 1989.

[24] A. S. FoKas AND Q. M. LiU, Asymptotic integrability of water waves, Phys. Rev. Lett., 77 (1996), pp. 2347-2351.

[25] Y. Kadama AND A. V. Mikhailov, Obstacles to asymptotic integrability, in Algebraic Aspects of Integrable Systems: in memory of Irene Dorfman, Progr. Nonlinear Differential Equations Appl. 26, A. S. Fokas and I. M. Gelfand. ed., Birkhäuser, Boston, 1997, pp. 173-204.

[26] A. Degasperis and M. Procesi, A test of asymptotic integrability of $1+1$ wave equations, preprint (1998).

[27] V. E. Zakharov and B. G. Konopelchenko, On the theory of recursion operator, Comm. Math. Phys., 94 (1984), pp. 483-509.

[28] P. M. SANTINI AND A. S. FOKAS, Recursion operators and bi-Hamiltonian structures in multidimensions. I., Comm. Math. Phys., 115 (1988), pp. 375-419.

[29] A. S. FoKAS AND P. M. SANTINI, Recursion operators and bi-Hamiltonian structures in multidimensions. II., Comm. Math. Phys., 116 (1988), pp. 449-474.

[30] R. M. MiURA, Korteweg-de Vries equation and generalizations. I. A remarkable explicit nonlinear transformation, J. Math. Phys., 9 (1968), pp. 1202-1204. 
